\title{
Questions and remarks to the Langlands $\operatorname{program}^{1}$
}

\author{
A. N. Parshin \\ (Uspekhi Matem. Nauk, 67(2012), n 3, 115-146; \\ Russian Mathematical Surveys, 67(2012), n 3, 509-539)
}

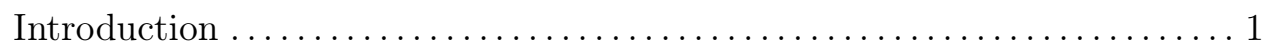

Basic fields from the viewpoint of the scheme theory $\ldots \ldots \ldots \ldots \ldots \ldots \ldots$

Two-dimensional generalization of the Langlands correspondence . . . . . 10

Functorial properties of the Langlands correspondence............... 12

Relation with the geometric Drinfeld-Langlands correspondence . . . . . . 16

Direct image conjecture .................................. 20

A link with the Hasse-Weil conjecture . . . . . . . . . . . . . . . . . . 27

Appendix: zero-dimensional generalization

of the Langlands correspondence ............................. 30

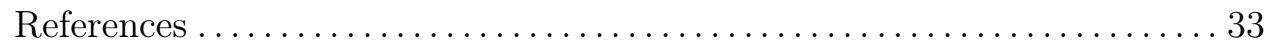

\section{Introduction}

The goal of the Langlands program is a correspondence between representations of the Galois groups (and their generalizations or versions) and representations of reductive algebraic groups. The starting point for the construction is a field. Six types of the fields are considered: three types of local fields and three types of global fields $[\mathbf{L} 3, \mathbf{F} 2]$. The former ones are the following:

1 ) finite extensions of the field $\mathbb{Q}_{p}$ of $p$-adic numbers, the field $\mathbb{R}$ of real numbers and the field $\mathbb{C}$ of complex numbers,

2) the fields $\mathbb{F}_{q}((t))$ of Laurent power series, where $\mathbb{F}_{q}$ is the finite field of $q$ elements,

3) the field of Laurent power series $\mathbb{C}((t))$.

The global fields are:

4) fields of algebraic numbers (= finite extensions of the field $\mathbb{Q}$ of rational numbers),

\footnotetext{
${ }^{1}$ I am grateful to R. P. Langlands for very useful conversations during his visit to the Steklov Mathematical institute of the Russian Academy of Sciences (Moscow, October 2011), to Michael Harris and Ulrich Stuhler, who answered my sometimes too naive questions, and to İhan İkeda who has read a first version of the text and has made several remarks. The work was supported by the RFBR grants 1107.0408, 1012.0486 and NSh-5139.2012.1.
} 
5) fields of algebraic functions in one variable with a finite constant field (= finite extensions of the field $\mathbb{F}_{q}(x)$ ),

6 ) fields of algebraic functions in one variable with the field $\mathbb{C}$ as the constant field ( $=$ finite extensions of the field $\mathbb{C}(x)$ ).

The local fields in the first list are completions of the global fields in the second one.

In the classical Langlands program, only the first two types of local and global fields are considered. The program, in its simplest (and most studied) variant, consist in the construction of a correspondence between finite dimensional (of dimension $n$ ) representations of the Galois group $G_{K}=$ $\mathrm{Gal}\left(K^{\text {sep }} / K\right)$ of a separable closure of the field $K$ and irreducible infinitedimensional (usually) representations of the group $G L\left(n, A_{K}\right)$, where $A_{K}=$ $K$ for the local fields $K$ and $A_{K}=\mathbb{A}_{K}$ (ring of adeles) for the global fields $K$.

Recall that $\mathbb{A}_{K}=\left\{\left(f_{v}\right)\right.$ : for all $v f_{v} \in K_{v}$, for almost all $\left.v f_{v} \in \hat{\mathcal{O}}_{v}\right\}$. Here $v$ runs through all classes of valuations of the field $K$ with complete valuation $\operatorname{rings} \hat{\mathcal{O}}_{v}$ and local fields $K_{v}=\operatorname{Frac}\left(\hat{\mathcal{O}}_{v}\right) \supset K$ which are the local completions of $K$. In the number field case, one has to add finitely many archimedean valuations of the field $K$ and the related embeddings of $K$ into the fields $\mathbb{R}$ or $\mathbb{C}$.

Globally, the representations that we consider here must be automorphic, i.e. realized in a space of functions on the quotient space $\operatorname{GL}\left(n, \mathbb{A}_{K}\right) / \operatorname{GL}(n, K)$. Since $n$-dimensional representations of the Galois group $G_{K}$ can be viewed as homomorphisms from $G_{K}$ in $\operatorname{GL}(n, \mathbb{C})$, then there is a natural generalization of this correspondence, when the group $\operatorname{GL}(n)$ is replaced by an arbitrary reductive algebraic group $G$. In this more general situation, the hypothetical Langlands correspondence should look like (in a first approximation) as follows

$$
\operatorname{Hom}\left(G_{K},{ }^{L} G(\mathbb{C})\right) \Leftrightarrow \quad \begin{gathered}
\text { irreducible (automorphic) } \\
\text { representations of the group } \left.G\left(A_{K}\right)\right\}
\end{gathered}
$$

Here ${ }^{L} G$ is a reductive algebraic group, Langlands dual group to $G$ (see the definition and basic properties in $[\mathbf{T} 2, \mathbf{C}]$ ). It is important and highly nontrivial that if the original group $G$ is regarded as a reductive group over the different fields in the basic list, then the dual group ${ }^{L} G$ is always a group defined over the complex numbers ${ }^{2}$. The group ${ }^{L} G$ contains the connected component of the identity ${ }^{L} G_{0}$, which is a reductive algebraic group over $\mathbb{C}$. Its root system is constructed by application of duality of tori, starting

\footnotetext{
${ }^{2}$ Or another field such as the field $\overline{\mathbb{Q}}_{l}$ which has nothing common with the ground field $K$.
} 
from the root system of the original group $G$. In addition, for the general linear group $G=\mathrm{GL}(n)$ the root systems of $G$ and ${ }^{L} G$ coincide. Thus, in this case, the presence of the duality is masked. Finally, the whole group ${ }^{L} G$ is a semidirect product of groups ${ }^{L} G_{0}$ and the Galois group $\operatorname{Gal}\left(K^{\text {sep }} / K\right)$.

The representations of Galois groups included in the LHS of the Langlands correspondence require substantial generalization, so that one can hope for the existence of bijections with automorphic representations of reductive groups that are on the RHS ${ }^{3}$. In the theory of Artin $L$-series there are considered representations of finite Galois groups with values in the group $\mathrm{GL}(n, \mathbb{C})$. If one goes to the Galois group $G_{K}$ of a separable closure of the ground field $K$, then all of its continuous representations in the group $\mathrm{GL}(n, \mathbb{C})$ are obtained from representations of finite factors $\operatorname{Gal}(L / K)$, where $L / K$ runs through all the normal finite extensions of $K$. Thus, there are not too many continuous complex representations of the Galois groups. On the other hand, there are continuous $l$-adic representations arising from Galois action on etale cohomology of algebraic varieties defined over $K$. They usually do not pass through the finite factors of Galois groups ${ }^{4}$. Deligne has invented how to build an extension of the Galois group $G_{K}$, whose complex representations "coincide"with the $l$-adic representations of $G_{K}$. The Weil-Deligne group $W D_{K}$ is a semidirect product of the Weil group $W_{K}{ }^{5}$ with $\mathbb{C}$ and the action $w z w^{-1}=q^{-\nu(w)} z$. Just,

$$
W D_{K}=\left\{w, z: w \in W_{K}, z \in \mathbb{C}\right\}
$$

and

$$
(w, z) \cdot\left(w^{\prime}, z^{\prime}\right)=\left(w w^{\prime}, z+q^{-\nu(w)} z^{\prime}\right)
$$

where $\nu: W_{K} \rightarrow \mathbb{Z}$ is the canonical mapping of the Weil group that sends $n$-th degree of the Frobenius automorphism onto $n$ and $q$ is the number of elements in the residue field ${ }^{6}$.

\footnotetext{
${ }^{3}$ We note that existence of the bijection was only assumed for the group $G=\operatorname{GL}(n)$. Generally, the correspondence may be not one-to-one and can have certain finite sets as preimages, i.e. so called $L$-packets.

${ }^{4}$ This is so if all cycles of the variety are algebraic. By the Tate's conjectures, the converse should be true.

${ }^{5}$ The Weil group $W_{K}$ of a local field $K$ with finite residue field is the subgroup of the Galois group $G_{K}$, consisting of those elements which act on the residue field through integer powers of the Frobenius automorphism.

${ }^{6}$ See details in [T2][n 4.1]. Instead of the Weil-Deligne group one can consider representations of the Weil-Arthur group $W A_{K}=W_{K} \times \mathrm{SL}(2, \mathbb{C})$. The transition from representations of one group to the representations of another one provided by the theorem of Jacobson-Morozov (see [L3]).
} 
In particular, the Langlands correspondence $\mathrm{LC}_{n}$ for local fields and the group $\mathrm{GL}(n)$ must have the form:

$$
\operatorname{Hom}^{c, s s}\left(W D_{K}, \mathrm{GL}(n, \mathbb{C})\right) \Leftrightarrow \quad \begin{gathered}
\text { ssmooth irreducible } \\
\text { representations of the group } \operatorname{GL}(n, K)\}
\end{gathered}
$$

and this is the final exact form (the representations of the Weil-Deligne group supposed to be continous and completely reducible). Smoothness of a representation $\pi: \operatorname{GL}(n, K) \rightarrow \operatorname{End}(V)$ means that for every $v \in V$ there is an open compact subgroup $\mathbb{K}^{\prime}$ in $\operatorname{GL}(n, K)$ such that $\pi\left(\mathbb{K}^{\prime}\right)(v)=v$. Irreducibility means the absence of any non-trivial invariant subspaces of $V$. Let $\rho, \rho^{\prime}$ run over $n$-dimensional representations of the Weil-Deligne group, and $\pi$ be a representation of $\operatorname{GL}(n, K)$. The Langlands correspondence has the following properties:

i) $\mathrm{LC}_{1}($ Det $\rho)=$ central character of the representation $\mathrm{LC}_{n}(\rho)$;

ii) $\mathrm{LC}_{n}(\rho \otimes \chi)=\mathrm{LC}_{n}(\rho) \otimes\left(\mathrm{LC}_{1}(\chi) \circ\right.$ Det $)$, where $\chi$ is a one-dimensional representation and Det $\mathrm{GL}(n, K) \rightarrow K^{*}$ is the determinant;

iii) $\operatorname{LC}_{m+n}\left(\rho \oplus \rho^{\prime}\right)=\operatorname{Ind}_{P}^{\mathrm{GL}(m+n, K)} \circ \operatorname{Res}_{M}^{P}\left[\operatorname{LC}_{m}(\rho) \otimes \mathrm{LC}_{n}\left(\rho^{\prime}\right)\right]$;

iv) if the representation $\rho$ is irreducible then representation $\operatorname{LC}_{n}(\rho)$ is a cuspidal one;

v) $\operatorname{LC}_{n}(\check{\rho})=\check{\operatorname{LC}_{n}}(\rho)$;

vi) $L(\rho, s)=L\left(\mathrm{LC}_{n}(\rho), s\right)$;

vii) if the representation $\rho$ is unramified then representation $\operatorname{LC}_{n}(\rho)$ is a spherical one.

For the property iii), we have used the embedding of the product of groups $\mathrm{GL}(m, K)$ and $\operatorname{GL}(n, K)$ into the group $\mathrm{GL}(m+n, K)$ as the Levi quotient $M=\mathrm{GL}(m, K) \times \mathrm{GL}(n, K)$ of the standard parabolic subgroup $P$. Concerning the operation $\otimes$ see note. 18 . The subsequent operation is parabolic induction which extends the representation of the Levi subgroup $M$ to the subgroup $P$ and then induces it to the whole group $\mathrm{GL}(m+n, K)$ (for details, as well as for the definition of cuspidal representations, see $[\mathbf{B Z}, \mathbf{C}]$ and $[\mathbf{B}][$ ch. 4] for the case $\mathrm{GL}(2))$.

Furthermore, $\check{\rho}(\check{\pi})$ denote dual (contragradient) representations arising from the representations $\rho(\pi)$. Let $L(\rho, s)$ be the Artin $L$-function of representation $\rho$ (see [T2] [n 3], [B][ch. 1.8]) and $L(\pi, s)$ be $L$-function of representation $\pi$ 
(see section on the Hasse-Weil conjecture ). A representation $\pi$ of $\mathrm{GL}(n, K)$ on a space $V$ is called spherical, if there exists a vector $v \in V$, such that $\pi(\mathbb{K})(v)=v$, where $\mathbb{K}=\operatorname{GL}\left(n, \mathcal{O}_{K}\right)$ is a maximal compact subgroup. The list of properties can be further supplemented by an equality for local constants appearing in the functional equation of $L$-functions (see $[\mathbf{H}]$ ).

Similar properties should hold for the global Langlands correspondence.

Up to now, the Langlands correspondence was constructed exactly in this form for the local fields of type 1) (M. Harris-R. Taylor [HT]; G. Henniart $[\mathbf{H}]$ ) and type 2) (G. Laumon-M. Rappoport-U.Stuhler [LRS]). For the global fields of type 5) and the group $G=\mathrm{GL}(n)$, the correspondence was obtained for $n=2$ by V. G. Drinfeld [Dr1, Dr2] and for arbitrary $n$ by L. Lafforgue $[\mathbf{L}]$. In any case, construction of the correspondence in the number field case remains an open question, and apparently very far from being resolved.

Previous constructions have a purely arithmetic origin. Based on his proof, which is concentrated around the moduli space of vector bundles on algebraic curves, Drinfeld has expanded the class of fields and introduced the geometric Langlands correspondence, which should take place for the fields of types 3) and 6).

The initial object will then be a complete algebraic curve $X$, defined over $\mathbb{C}$, and for which the field $K$ is a field of rational functions on $X$. The correspondence looks as follows

$$
\text { Local }{ }^{L} G \text { - systems on } X \Leftrightarrow \text { Hecke sheaves on scheme (stack) Bun }{ }_{X, G}
$$

and was proven for unramified local systems and the group $G=\operatorname{GL}(n)$ (V. G. Drinfeld for $n=2$ and E. Frenkel - D. Gaitsgory - K. Vilonen for any $n$, based on a construction of G. Laumon). Since local (unramified) ${ }^{L} G$ system on $X$ are nothing but a representation of the fundamental group of $X$ in the group ${ }^{L} G$, it looks as a quite natural analog of the arithmetical situation. On the other hand, there is $B u n_{X, G}$, the stack of principal $G$-bundles on $X$, and the ring of Hecke correspondences acts on it. For the stack $B u n_{X, G}$, one needs not the usual local systems but perverse sheaves which are considered instead of automorphic forms in the classical Langlands correspondence. This sheaf is called the Hecke sheaf, if it is semi-invariant with respect to Hecke correspondences. In this situation, the Hecke sheaves do not replace the representations of the adelic group responsible, according to the arithmetic Langlands correspondence, to the representations of the Galois group, but they correspond to the spherical vectors of these representations. Whether there are the representations itself, I do not know, but, if they exist, they should be in a reasonable sense unramified (spherical)). In the arithmetical 
situation the spherical vector is uniquely defined, up to a constant, and generates the representation space.

What are the problems in number theory that can be studied with help of the Langlands program?

1. Non-abelian class field theory. Langlands correspondence arose from consideration of the abelian situation where $G=\mathrm{GL}(1)$ and the representation of the Galois groups are homomorphisms of $G_{K}$ into $\mathrm{GL}(1, \mathbb{C})=\mathbb{C}^{*}$, i.e. characters of $G_{K}$. Previously, such maps are called quasi-characters, keeping the term "character"for the homomorphisms in the unitary group $U(1) \subset$ $\mathrm{GL}(1, \mathbb{C})$. By class field theory we have the reciprocity map,

$$
C_{K} \rightarrow G_{K}^{a b}
$$

where $C_{K}=K^{*}$ for local fields of types 1) and 2) and $C_{K}=\mathbb{A}_{K} / K^{*}$ for global fields of types 4) and 5). Characters of $C_{K}$ (and they are automorphic forms on GL(1)) can be transferred to the group $G_{K}$ as its abelian characters, i.e. 1dimensional representations. We thus obtain an abelian Langlands correspondence [L2], which served as a sample and the starting point of the whole program.

However, there is a fundamental difference between the general Langlands construction and its abelian case. Actually, the difference is related to the problem of explicit description of the Galois group. Class field theory allows to compute explicitly the Galois group of the maximal abelian extension of the original field (specifying its generators and relations). Moreover, the cohomological formulation of class field theory makes it possible to compute the Galois group of some non-abelian extensions ( $l$-extensions [Sh, Koch]).

The Langlands program allows us to describe the set of representations of the Galois group, providing it with a standard set of operations (primarily direct and tensor product). Further development of the program was to construct a category of representations of the Galois group as a monoidal category (more precisely, the Tannaka category or, probably, its generalization, see discussion in [L3, Ram]), which allows to reconstruct uniquely the group (in view of a general theorem of the theory of Tannaka categories $[\mathbf{B r}]$ ). Note that this theorem is an existence theorem and it is unclear how to get an explicit presentation through generators and relations.

2. Conjectures on zeta- and $L$-functions of arithmetical schemes (see below the section on the Hasse-Weil conjecture). In this direction, a great achievement is the proof of Artin's conjecture on the holomorphy of $L$-series for a wide class of two-dimensional representations of the Galois group over $\mathbb{Q}$ (R. P. Langlands-J. Tunnell). Actually, a special case of the octahedral representations was a starting point for the A. Wiles proof of the Taniyama-Weil conjecture. 
3. Description of the set of smooth irreducible representations of reductive groups over local and global fields.

The last line is the most successful. We have seen how the point of view of representation theory of reductive groups requires certain changes of the Galois group on the Weil-Deligne group or the Weil-Arthur group. This is most sharply shown by the example of a local field $K=\mathbb{C}$. The group $\mathrm{GL}(n, K)$ has for $K=\mathbb{C}$ a nontrivial set of irreducible representations (all of them are the principal series representations and their parameter space is a complex manifold $=$ quotient-space of the manifold of characters of torus $\left(\mathbb{C}^{*}\right)^{n}$ by the permutation group $\left.S_{n}{ }^{7}\right)$. At the same time Galois group $G_{K}$ is trivial, but the Weil group is, according to the general theory [AT][ch. $\mathrm{XV}],[\mathbf{T} 2][\mathrm{n} 1], W_{\mathbb{C}}=\mathbb{C}^{*}$ and has exactly the same space of $n$-dimensional semisimple representations $[\mathbf{K n}]$.

We now consider the basic fields of the Langlands program in terms of general principles of arithmetic algebraic geometry.

\section{Basic fields from the viewpoint of the scheme theory}

The fields, considered in the Langlands' theory, are fields of functions on some schemes. In the scheme theory, we have a classification by a dimension. If the scheme is affine and corresponds to a ring $A$, then we can take its Krull dimension. We have the following table of rings, arising in arithmetic:

\begin{tabular}{|c|c|c|}
\hline $\operatorname{Dim}(A)$ & geometric case & number case \\
\hline$>2$ & $\ldots$ & $\ldots$ \\
\hline 2 & $\begin{array}{c}\mathbb{F}_{q}[x, y] \\
\text { (surfaces) }\end{array}$ & $\begin{array}{c}\mathbb{Z}[y] \\
\text { (arithmetic surfaces) }\end{array}$ \\
\hline 1 & $\begin{array}{c}\mathbb{F}_{q}[x] \\
\text { (curves) }\end{array}$ & $\begin{array}{c}\mathbb{Z} \\
(\text { arithmetic curves, } \operatorname{Spec}(\mathbb{Z}))\end{array}$ \\
\hline 0 & $\mathbb{F}_{q}$ & $\mathbb{F}_{1}$ \\
\hline
\end{tabular}

Here, $\mathbb{F}_{q}$ is a finite field of $q$ elements, $\mathbb{Z}$ is the ring of integers and $\mathbb{F}_{1}$ is now popular field of one element.

These rings correspond to affine schemes and the fields of functions on them. In particular, this table includes global fields of types 4) and 5) from

${ }^{7}$ D. P. Zhelobenko-M. A. Naimark's theorem (1966, see exposition in [Zh]). Explicit form for the characters of the torus $\mathbb{C}^{*}$ and consequently torus $\left(\mathbb{C}^{*}\right)^{n}$ see below and more detailed in $[\mathbf{K n}]$. 
the list of fields that are now considered as the main ones for the Langlands program (see $[\mathbf{L} \mathbf{3}, \mathbf{F} 2])$ :

\begin{tabular}{|c|c|c|}
\hline $\operatorname{Dim}(A)$ & geometric case & number case \\
\hline 2 & $\begin{array}{c}\mathbb{F}_{q}(x, y) \\
\text { (rational functions) }\end{array}$ & $\begin{array}{c}\mathbb{Q}(y) \\
\text { (rational functions) }\end{array}$ \\
\hline 1 & $\mathbb{F}_{q}(x)$ & $\mathbb{Q}$ \\
(rational functions) & (rational numbers) \\
\hline 0 & $\mathbb{F}_{q}$ & $\mathbb{F}_{1}$ \\
\hline
\end{tabular}

Finally, there is a table of the local fields that arise under these circumstances:

\begin{tabular}{|c|c|c|}
\hline $\operatorname{Dim}(A)$ & geometric case & number case \\
\hline 2 & $\begin{array}{c}\qquad \mathbb{F}_{q}((x))((y)) \\
\text { (iterated Laurent power series) }\end{array}$ & $\begin{array}{c}\mathbb{Q}_{p}((y)), \quad \mathbb{R}((y)), \quad \mathbb{C}((y)) \\
\text { (Laurent power series) }\end{array}$ \\
\hline 1 & $\begin{array}{l}\qquad \mathbb{F}_{q}((x)) \\
\text { (Laurent power series) }\end{array}$ & $\begin{array}{c}\mathbb{Q}_{p}, \quad \mathbb{R}, \mathbb{C} \\
\text { (p-adique, real and complex } \\
\text { numbers })\end{array}$ \\
\hline
\end{tabular}

Here we see all types 1) - 3) of local fields, but also a number of the new fields which were introduced in the higher adelic theory $[\mathbf{P} 3]^{8}$. Such a classification is the development of ideas of Andre Weil from his report to the Cambridge congress in 1950 [W2], where he stressed the importance of the concept of the Krull dimension for the classification of arithmetic problems. In his pre-war letter to sister Simone [W1] where Weil also speaks on the analogy between the number and the geometric case, the situation is simplified, and so he adds the field $\mathbb{C}((y))$ and puts it on the "one line"with such fields as $\mathbb{F}_{q}((x)), \mathbb{Q}_{p}$, what as seen from the table is not consistent with the dimension.

Returning to the global fields, we may ask, where in these tables are such schemes as the curves over the complex numbers associated with fields of type 6)? These curves and the fields of functions on them are the subject of the geometric Langlands correspondence, generalizing the usual Langlands correspondence. Indeed, they are not here, as they correspond to the rings of the form $\mathbb{C}[x]$. These objects have an intermediate nature, they are global and of dimension 1 over $x$ (the coordinate on the curve), and local in the field of definition $\mathbb{C}$. This field is included in the table as a one-dimensional

\footnotetext{
${ }^{8}$ See their informal definition at the beginning of the following section.
} 
(!) local field. Thus, such a ring as $\mathbb{C}[x]$ correspond to a geometric object of dimension 2. That is, they are archimedean fibers of arithmetic surfaces (on them see below).

This observation and the fundamental arithmetic analogies $[\mathbf{P 2}]$ suggest to consider, as a "partner"of the geometric Langlands correspondence for curves over $\mathbb{C}$, constructions of this kind for curves over $p$-adic numbers $\mathbb{Q}_{p}$. As far as I know, this was not considered by anyone and later we'll discuss how it might look ${ }^{9}$.

Summarizing this discussion, we can say that the list of six basic fields could be completed as follows. Only four types of fields - 1), 2), 4), 5) belong to the one-dimensional situation. Fields of types 3) and 6) are already in a two-dimensional situation. In this case we have two types of local fields

1) finite field extensions of $\mathbb{Q}_{p}((t)), \mathbb{R}((t)), \mathbb{C}((t))$ and $\mathbb{Q}_{p}\{\{t\}\}^{10}$.

$2)$ the field of Laurent power series $\mathbb{F}_{q}((u))((t))$.

and two types of global fields:

3 ) the fields of functions on arithmetic surfaces (= finite extensions of the field $\mathbb{Q}(x)$ of rational functions).

4) the fields of functions on algebraic surfaces over a finite field of constants (= finite extensions of the fields $\mathbb{F}_{q}(x, y)$ of rational functions in two variables).

Between them lie the fields of intermediate types (partially local, partially global). In the situation of a surface over a finite field the intermediate fields are

2a) finite extensions of the fields $\mathbb{F}_{q}(u)((t))$.

$2 \mathrm{~b})$ the field of fractions of the ring $\mathbb{F}_{q}[[u, t]]$ of formal power series in two variables.

In the case of arithmetic surfaces, the field $\mathbb{C}(x)$ (the field 6) from the list of Langlands) is also an intermediate field but it is not included in the standard set of fields appearing in the two dimensional adelic theory (see below and $[\mathbf{P 3}]$ ). The reason is that it due to the additional structure: the existence of a morphism from the two-dimensional scheme onto a onedimensional scheme $B$ and its fibers, defined over one-dimensional local fields of the scheme $B$.

Remark 1. Adelic technique allows us to consider local and global fields

\footnotetext{
${ }^{9}$ As was pointed out to me by İlhan İkeda, a construction of this kind was recently found by A. G. M. Paulin for abelian case.

${ }^{10}$ If $K$ is locally compact field with a non-Archimedean norm $|\cdot|$, then the field $K\{\{t\}\}$ consists of the infinite in both directions series $\sum_{i} a_{i}, a_{i} \in K$ with $\left|a_{i}\right| \leq O(1),\left|a_{i}\right| \rightarrow 0$ for $i \rightarrow-\infty$. The fields $\mathbb{Q}_{p}\{\{t\}\}$ can arise on the arithmetic surfaces.
} 
of the original Langlands program in a simultaneous way. As we have seen, in both cases, these fields are the fields of functions on a scheme $X$. In the local (non-Archimedean!) case we have $X=\operatorname{Spec}(\mathcal{O})$, where $\mathcal{O}$ is a local discrete valuation ring with finite residue field. In global situation, $X$ is either a curve over a finite field or spectrum of the ring of an algebraic number field. In both cases, $A_{K}=\mathbb{A}_{X}$ if we apply the general definition of the ring of adeles [FP, P3], which may include and the archimedean components as well.

\section{Two-dimensional generalization of the Langlands correspondence}

We may wander about existence of a two-dimensional generalization of the Langlands correspondence. Let $X$ be a two-dimensional scheme and $K$ be the field of functions on it. Then the $n$-dimensional representations of the Galois group $G_{K}$ of a separable closure for $K$ could correspond to irreducible automorphic representations of the adelic group $G\left(\mathbb{A}_{X}\right)$. Here, the ring of adeles $\mathbb{A}_{X}$ was introduced in the higher adelic theory in the 1970 s (see surveys [FP, P3]).

If $x \in C \subset X$ is a flag on $X$, consisting of a point $x$ and an irreducible curve $C$, and the point $x$ is smooth on $C$ then one can introduce a twodimensional local field $K_{x, C}$. In local coordinates $u, t$ of a formal neighborhood of the point $x$, the field $K_{x, C}$ is equal to $k(x)((u))((t))$ (if $X$ is a smooth surface), where $C=(t=0)$ and $k(x)$ is the residue field of $x$. In general, one can attach a direct sum of finitely many two-dimensional local fields to the flag $x, C$. The adelic ring $\mathbb{A}_{X}$ is defined as the adelic (a part of the total) product of the fields $K_{x, C}$ for all flags $x, C$.

The field $K_{x, C}$ contains a discrete valuation $\operatorname{ring} \hat{\mathcal{O}}_{x, C}($ which is $k(x)((u))[[t]]$ if $X$ is a surface). For an irreducible curve $C$, we take $K_{C}=\operatorname{Frac}\left(\hat{\mathcal{O}}_{C}\right)$ and

for a point $x$, we set $K_{x}=K \hat{\mathcal{O}}_{x} \subset \operatorname{Frac}\left(\hat{\mathcal{O}}_{x}\right)$. Here, $\hat{\mathcal{O}}_{C}=k(C)((t))$ and $\hat{\mathcal{O}}_{x}=k(x)[[u, t]]$. The adelic product of these rings give rise to three subrings $\mathbb{A}_{12}, \mathbb{A}_{01}, \mathbb{A}_{02}$ in $\mathbb{A}_{X}$. These rings are embedded into the $\mathbb{A}_{X}$ in a diagonal way (exactly as the principal adeles in the case of one-dimensional schemes). We can compare the structure of the local adelic components in dimensions one and two:

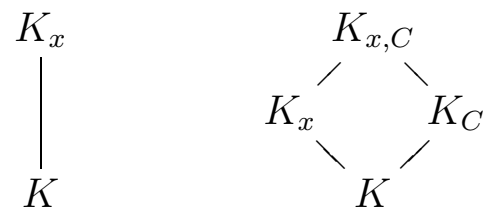

A generalization of the Langlands program that uses this theory of adeles, 
were outlined by M. Kapranov $[\mathbf{K}]$ (and detailed in $[\mathbf{I}]$ ). It uses not ordinary notion of representation, but its generalization associated with 2-categories $[\mathbf{G K}]$.

Here the number 2 stands as dimension of the scheme $X$. We have the following table of categories:

\begin{tabular}{|c|c|c|c|}
\hline$n=\operatorname{dim}$ & $n$-categories & objects & $\begin{array}{c}\text { distinguished } \\
\text { object }\end{array}$ \\
\hline 2 & 2 -Vect $/ k$ & Vect-mod $/ k$ & Vect $/ k$ \\
\hline 1 & Vect $/ k$ & $V / k$ & $k$ \\
\hline 0 & $k$ & $A \subset k$ & $\{1\}$ \\
\hline
\end{tabular}

where let $k$ be a ground field (or a ring), $A$ be its subset, $V$ be vector spaces over $k$ and Vect/ $k$ be the category of all vector spaces over $k$. The latter is a tensor category, and it can be considered as a categorical generalization of the notion of ring. Then, we can define a category of modules Vect-mod/ $k$ over such a "ring"and they form a 2-category whose objects are such categories.

We see an obvious inductive structure that can be easily compared with inductive structure of the $n$-dimensional local fields. In addition, if there is a group $G$, then we can introduce a concept of $n$-representation. 1-representation is the usual homomorphism in the group of automorphisms of a vector space. 2-representations are obtained replacing the spaces by categories and the homomorphisms by functors. More precisely, instead of the space $V$ we choose the category module Vect-mod/ $k$ and the 2-representation $\pi$ is to attach to an element $g$ of $G$ a functor $\pi(g): \operatorname{Vect-mod} / k \rightarrow \operatorname{Vect-mod} / k$, satisfying a condition of multiplicativity. This generalization is a special example of the general categorification process of concepts and structures ${ }^{11}$.

Then, in a certain approximation the Langlands correspondence for twodimensional local field $K$ looks, according Kapranov, as follows:

$$
\begin{array}{ccc}
\{n \text {-dimensional complex (or } l \text {-adic }) & \Leftrightarrow \quad \text { irreducible 2-representations } \\
\text { Galois representations of } \left.G_{K}\right\} & \text { of the group } \operatorname{GL}(2 n, K)\}
\end{array}
$$

and for a global field $K$ on the scheme $X$ the field $K$ in the RHS side of the correspondence is replaced by the ring $\mathbb{A}_{X}$ of adeles. The 2-representation must satisfy a certain automorphic condition. These definitions and related concepts need to be clarified and one has to understand certain restrictions they hold. In particular, the characteristic of the basic field is supposed to be 0 and nothing suggested for the local fields of archimedean type. Surely,

\footnotetext{
${ }^{11}$ The 0-representations will be discussed later in appendix.
} 
the construction has to exist for these fields and for the fields of finite characteristic. We shall return to this issue ${ }^{12}$.

\section{Functorial properties of the Langlands correspondence}

The usual Langlands correspondence has a large number of functorial properties. The theory includes two kinds of objects: a scheme or a field and a reductive algebraic group (better to say, a root data). The Langlands principle of functoriality [L3] says what happens when one changes the group. We will restrict ourselves here with the properties related to a change of the schemes ${ }^{13}$. First, they are the properties associated with finite extensions of fields (base change and automorphic induction or lifting). Second, the properties associated with the transition from local to global fields. All these properties are special cases of the general functorial construction of the inverse and direct images.

The first properties related to finite surjective morphisms of one-dimensional schemes $f: X \rightarrow Y$, where both $X, Y$ are either curves (or rings of integers in algebraic number fields) or the spectrum of local rings. The latter related to morphisms $f: X \rightarrow Y$, where $X$ is a spectrum of a local ring, and $Y$ is a curve. Let $B$ be a curve over $\mathbb{F}_{q}$ and $b \in B$. Then there is the commutative diagram

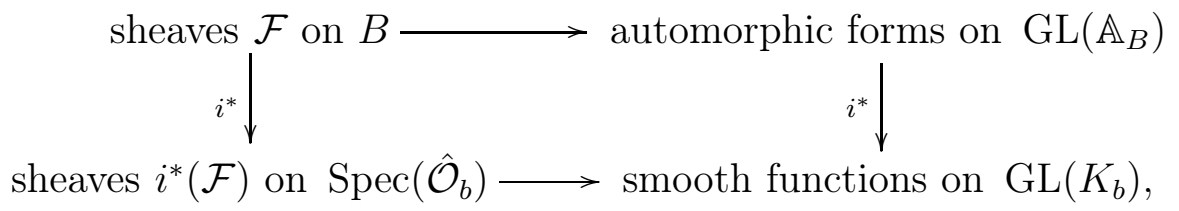

where $i: \operatorname{Spec}\left(\hat{\mathcal{O}}_{b}\right) \rightarrow B$ is the canonical embedding, the left map $i^{*}$ is the inverse image of sheaves and the right map $i^{*}$ is taking of the local $b$ component of a function on the whole adelic group (the non-trivial examples of this diagram see in $[\mathbf{H T}]$ ).

If the Langlands correspondence exists in the one form or another for the higher dimensions, it should have a much greater number of functorialities. Indeed, let a two-dimensional scheme $X$ be considered together with a structure morphism $f: X \rightarrow B$, where $B$ is an one-dimensional scheme.

Let us assume for simplicity that our schemes are varieties defined over

\footnotetext{
${ }^{12}$ Recently, D. V. Osipov, developing the ideas of Kapranov, proposed a definition of unramified local Langlands correspondence for two-dimensional local fields and the group $\mathrm{GL}(n)$ for any $n[\mathbf{O 2}]$.

${ }^{13}$ These two are not independent. In fact, the functoriality principle implies the automorphic induction and base change for finite extensions.
} 
a finite field. For $l$-adic sheaves (representations of the Galois groups), there are direct (pushforward) and inverse (pullback) images under the mapping $f$. Then, by Langlands correspondence, the same kind of operations should exist for representations of adelic groups.

Without discussing this question in full generality, let us consider a special and already extremely interesting case. Let us start with an one-dimensional representation of the Galois group on $X$. It determines certain sheaf $\mathcal{F}$ of rank 1 on $X$ and the direct images $R^{i} f_{*}(\mathcal{F})$ on $B, i=0,1,2$.

In this abelian situation on the surface $X$, there is already the Langlands correspondence between characters of the Galois group $G_{K}$ and characters of the group $K_{2}\left(\mathbb{A}_{X}\right)$. By the two-dimensional class field theory (see surveys $[\mathbf{F P}, \mathbf{I n v}, \mathbf{R}])$, there is a canonical map

$$
\varphi_{X}: K_{2}\left(\mathbb{A}_{X}\right) \longrightarrow G_{K}^{a b}
$$

which gives the map of groups of characters in the opposite direction. The reciprocity laws on the $X$ read as

$$
\varphi_{X}\left(K_{2}\left(\mathbb{A}_{01}\right)\right)=(1), \varphi_{X}\left(K_{2}\left(\mathbb{A}_{02}\right)\right)=(1) .
$$

We can assume that on the "automorphic side" of the Langlands correspondence there are also such operations as direct and inverse images between the characters of $K_{2}\left(\mathbb{A}_{X}\right)$ and automorphic forms on $\mathbb{A}_{B}$.

The case of the inverse image is not hypothetical, but follows from the known results of the higher adelic theory. There is an operation of the direct image for abelian groups $[\mathbf{K a}, \mathbf{O} \mathbf{1}]^{14}$.

$$
f_{*}: K_{2}\left(\mathbb{A}_{X}\right) \rightarrow K_{1}\left(\mathbb{A}_{B}\right) .
$$

Since $K_{1}\left(\mathbb{A}_{B}\right)=\mathrm{GL}\left(1, \mathbb{A}_{B}\right)$, we obtain a map of automorphic forms on $\mathrm{GL}\left(1, \mathbb{A}_{B}\right)$ to the automorphic forms, that is, characters on $K_{2}\left(\mathbb{A}_{X}\right)$. Their "automorphy"will be justified by higher reciprocity laws on the scheme $X$.

\footnotetext{
${ }^{14}$ In this paper, the map $f$ is smooth. The analogous homomorpism for differential forms was constructed in $[\mathbf{M}]$ for non-smooth maps. Quite recently [Liu], the direct image morphism of the $K$-groups was built for non-smooth maps of algebraic and also arithmetic surfaces.
} 
We have the commutative diagram

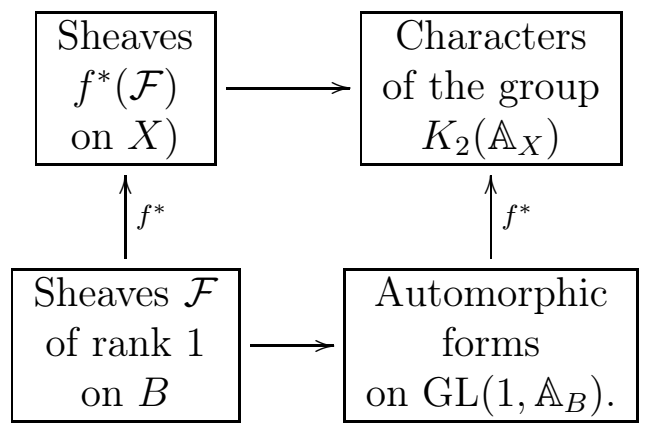

The direct image must ${ }^{15}$ consist in a construction of automorphic forms on $\operatorname{GL}\left(r_{i}, \mathbb{A}_{B}\right)$, corresponding to the characters of $K_{2}\left(\mathbb{A}_{X}\right)$ on $X$. The following commutative diagram should be true:

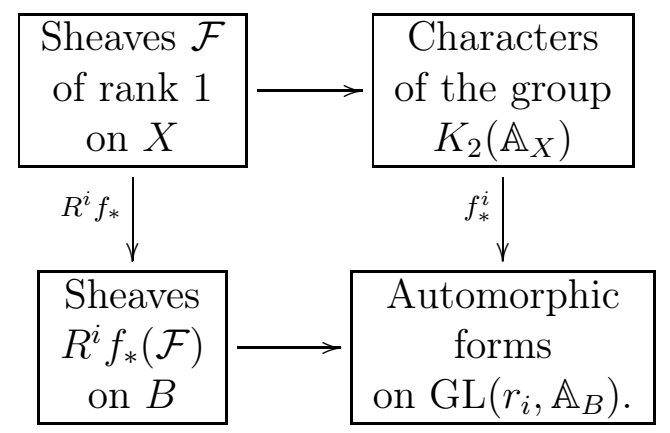

Here $r_{i}$ is rank of the sheaf $R^{i} f_{*}(F)$. It is quite possible that instead of the groups $\mathrm{GL}\left(r_{i}, \mathbb{A}_{B}\right)$ separately, there must be considered "even" $\mathrm{GL}\left(2, \mathbb{A}_{B}\right) \sim$ $\mathrm{GL}\left(r_{0}, \mathbb{A}_{B}\right) \times \mathrm{GL}\left(r_{2}, \mathbb{A}_{B}\right)$ and "odd" $\mathrm{GL}\left(r_{1}, \mathbb{A}_{B}\right)$ groups. Also, in the case of "middle"group, $i=1$, the monodromy preserving the skew-symmetric cup product could be taken into account and possibly one has to consider the orthogonal group corresponding to symplectic group (of the Galois action) according to the $L$-duality ${ }^{16}$.

Remark 2. We may compare this picture with a number field case where a two-dimensional regular scheme $X$ is mapped on a one-dimensional scheme

\footnotetext{
${ }^{15} \mathrm{We}$ deliberately describe this hypothetical construction in a preliminary vague form. Generally, the Langslands correspondance includes the irreducible automorphic representations on the RHS. Nevertheless, in many cases it is possible to find a generating vector in the spaces of representations. These vectors lead to automorphic forms on the adelic groups. So, for an unramified case there are spherical vectors and unramified automorphic functions; for generic representations one can use the Whittaker functions. At the end of this note we give an exact formulation in a simple but still non-trivial case.

${ }^{16}$ More exactly, the group $\operatorname{GSpin}(2 g+1, \mathbb{C})$ which is $L$-dual to the group $\operatorname{GSp}(2 g)$ of simplectic similitudes.
} 
$B$ (= the ring of integers in a number field). We expect the same kind of construction also in this case. If the general fiber of the mapping $f$ is an elliptic curve over $\mathbb{Q}, B=\operatorname{Spec}(\mathbb{Z})$ and $\chi$ is trivial, then the automorphic form $f_{*}^{1}(\chi)$ is nothing but an adelic version of the parabolic form corresponding to the curve according to the Taniyama-Weil conjecture (now a theorem!). In the Langlands program, this form generates a cuspidal representation of the group $\mathrm{GL}\left(2, A_{B}\right)[\mathbf{L} \mathbf{1}, \mathbf{G}]$. At the same time, the less complicated representation associated (in our notation) with forms $f_{*}^{0}(\chi)$ and $f_{*}^{2}(\chi)$ somehow remained in the shadows. These forms are defined on the group $\mathrm{GL}\left(1, \mathbb{A}_{B}\right)$. Thus, they are characters of $\mathbb{A}_{B}^{*}$ and, therefore, they give a character $\eta$ of the standard maximal torus $T$ in $G=\mathrm{GL}\left(2, \mathbb{A}_{B}\right)$. Parabolic induction applied to $\eta$ (if it is not equal to 0 ) gives an induced representation from the principal series of $G$ which is generated by an Eisenstein series. Thus, the original character $\chi$ can be associated with two infinite-dimensional representations of $\operatorname{GL}\left(2, \mathbb{A}_{B}\right)$, the "even"one belonging to the continuous spectrum, and the other "odd"one belonging to the discrete spectrum.

Return back to our diagram. Since we consider the case of curves over a finite field the correspondence of the bottom line was constructed (DrinfeldLafforgue) and one "only"needs to close the entire diagram. It seems natural to build first the local and semi-local direct images that enter into the following diagram

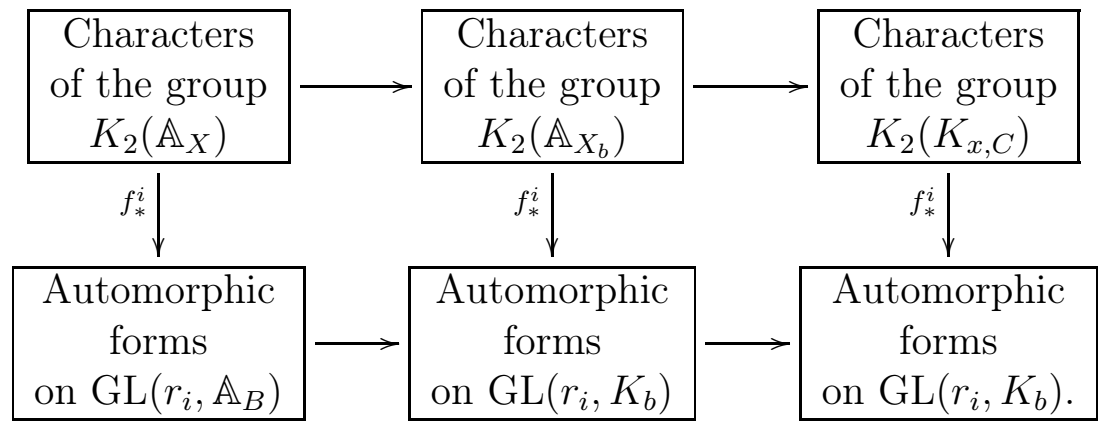

Here, $b=f(x), C$ is a curve on $X$, passing through $x$ (in particular, the fiber $F_{b}$ of the map $\left.f\right), X_{b}$ is the two-dimensional scheme, $X_{b}=X \times{ }_{B} \operatorname{Spec}\left(\mathcal{O}_{b}\right)=$ infinitesimal neighborhood of the fiber $f^{-1}(b)$.

The next step should be to prove the global automorphic property for the image-form on $B$, starting from the reciprocity laws for the character to $K_{2}\left(\mathbb{A}_{X}\right)$. Note that these issues can be considered completely independent of how one can arrange the Langlands correspondence for sheaves $\mathcal{F}$ of rank $>$ 1 on $X$.

Also, it is important to consider functorial properties of the Langlands correspondence for one-dimensional schemes (conventional theory) and schemes 
(= finite fields) of dimension 0. This question was raised by me in the late 1970s and discussed then with A. Beilinson. Although it was clear that the correspondence in dimension 0 must be something simple, we could not think it out. When M. Kapranov sent me the first version of his work, I suggested that he has to find this definition and add it to his two-dimensional construction and he did this in the final text. However, he did not consider functorial properties associated with surjective maps of the schemes of different dimensions.

In the case of schemes of dimension 1 and 0 , we have a diagram

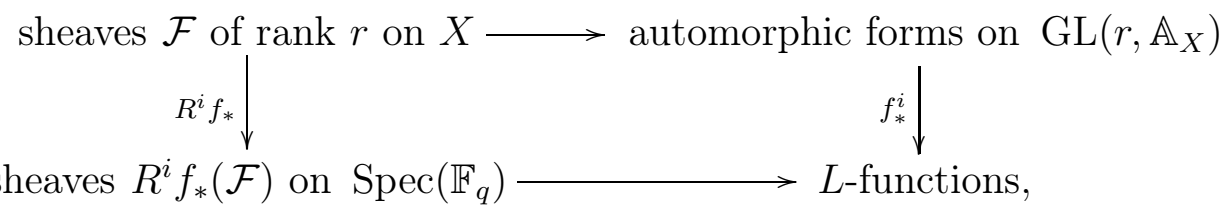

where $X$ is a curve over a finite field $\mathbb{F}_{q}$, sheaves on $\operatorname{Spec}\left(\mathbb{F}_{q}\right)$ are vector spaces with Frobenius action and $L$-functions $=$ characteristic polynomials of the Frobenius automorphisms. The lower line refers to zero-dimensional situation and is the Langlands correspondence for dimension zero according to Kapranov's proposal ${ }^{17}$.

This diagram should be consistent with the hypothetical diagram which we proposed above for a morphism of scheme $X_{b}$ on $\operatorname{Spec}\left(\mathcal{O}_{b}\right)$, if we take a closed fiber $F_{b}=X \times \operatorname{Spec}(k(b))$ as the curve $X$. The starting point of such concordance is the cartesian commutative diagram

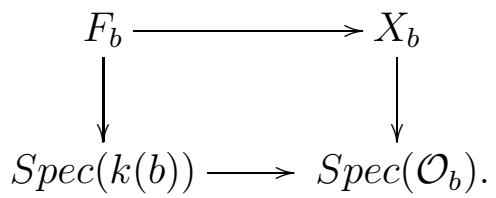

\section{Relation with the geometric Drinfeld-Langlands correspondence}

Let us now consider the same picture for the number field case where the scheme $X$ is mapped onto an one-dimensional scheme $B$ (= the ring of integers) and completed in accordance with the Arakelov theory by archimedean fibers $X \times_{B} \mathbb{C}$ or $X \times_{B} \mathbb{R}[\mathbf{P} 2]$.

Semi-local schemes $X_{b}$ in the new archimedean situation no longer exist, but we must find an analogue of the above structures and in this case as well (according to the general principles of analogy "number $\sim$ functions"in

\footnotetext{
${ }^{17}$ See a discussion of this proposal below in appendix.
} 
arithmetic [P2]). It would be natural to expect that such a two-dimensional (!) Kapranov's analogue of the Langlands correspondence for scheme $X \times{ }_{B} \mathbb{C}$ (so far only very hypothetical) is already formulated and partially proven geometric Drinfeld-Langlands correspondence on the curve $X$, defined over the complex field $\mathbb{C}$, and which appears here as an archimedean fiber. We will discuss this possibility later.

Let us assume now that in this situation, there are also direct images and the commutative diagram

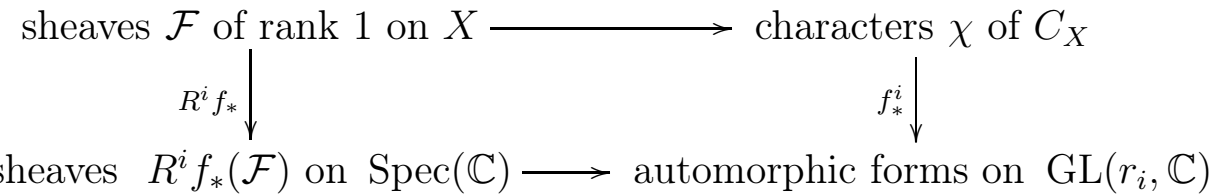

where $f: X \rightarrow \operatorname{Spec}(\mathbb{C}), C_{X}$ is a suitable class formation (see $[\mathbf{A T}]$ ) in the class field theory for the field $\mathbb{C}(X)$ of functions on $X$. Such formations are constructed together with class field theory for the fields of type $\mathbb{C}(X)$ in the 1950s by J.-P. Serre, M. Hasewinkel [S2, Has] and Y. Kawada-J. Tate $[\mathbf{K T}]$ . They are as follows,

$$
C_{X}=\pi_{1}\left(\mathbb{A}_{X}^{*(0)} / K^{*}\right)=\pi_{1}\left(\lim _{\longleftarrow} J_{m}(X)\right),
$$

where $(0)$ denotes the degree 0 ideles, $J_{m}(X)$ are generalized Jacobian varieties with "module" $m$ [S1], the limit is taken over all $m$ and it is a pro-algebraic group in the sense of Serre [S2].

The bottom line of the diagram is well known in the classical theory of the local Langlands correspondence for the field $\mathbb{C}$. "Sheaves"on $\operatorname{Spec}(\mathbb{C})$ are vector spaces of finite dimension over $\mathbb{C}$ equipped with a Hodge structure. Note that the cohomology of the local system (= sheaf) on $X$ can be equipped with a canonical Hodge structure. And it is this structure that defines a representation of the Weil group $W_{\mathbb{C}}$ for the field $\mathbb{C}$, which plays a role, in this situation, of the Galois group. We have $W_{\mathbb{C}}=R_{\mathbb{C} / \mathbb{R}} \mathbb{G}_{m}=\mathbb{C}^{*}$. So, there arises

Question 1. How to construct in an explicit way the direct image = the RHS arrow of the diagram?

But one first need to figure out what is the correct definition of the Langlands correspondence in this situation. As above, we can and must begin with the local Langlands correspondence, local on $X$. The existing Drinfeld theory (see a survey $[\mathbf{F 1}]$ ) consideres a local correspondence associated with 
the local fields of the form $\mathbb{C}\left(\left(t_{P}\right)\right)$ and that correspond to points $P$ of $X$ $\left(\right.$ Here,$\left.t_{P}\right)$ is a formal local parameter at the point $P$ ). The local correspondence must describe the $n$-dimensional representations of the group, which is a local version of the Galois group (or of the fundamental group in the unramified case). This is already interesting to study the case of an abelian group and $n=1$. Thus we arrive at the question about the local analogue of class field theory for such fields. Since they are archimedean variants of two-dimensional local fields, it is natural to ask:

Question 2. What is the relationship with the two-dimensional adelic class field theory?

If $K$ is a two-dimensional local field with finite last residue field, then the class field theory consists in a construction of the canonical reciprocity map,

$$
K_{2}(K) \longrightarrow G_{K}^{a b}
$$

which is a local component of the global map considered above. The kernel of this map coincides with the subgroup of divisible elements in $K_{2}(K)$ (I. Fesenko's theorem, see his survey in [Inv]). Examples of two-dimensional local fields are the fields $\mathbb{F}_{q}((u))((t)), \mathbb{Q}_{p}((t))$ and for them the class formations may be determined (in a very non-trivial way) and the Weil groups are defined as an extension of the Galois group by a group $K_{2}(K)[\mathbf{K o}, \mathbf{A}]$.

In the case of local field $\mathbb{C}((t))$ with archimedean residue field, we have the following structure of $K$-groups

$$
K_{2} \mathbb{C}((t)) \rightarrow K_{1} \mathbb{C}=\mathbb{C}^{*}
$$

where the group $\mathbb{C}^{*}$ is not the Galois group of $\mathbb{C}$, but its Weil group (!) and one may assume that the Weil group of the field $\mathbb{C}((t))$ can be defined by the same construction as above, making use of the group $K_{2} \mathbb{C}((t))$. This gives us the LHS part of the local abelian Langlands correspondence for $\mathbb{C}((t))$. The question of the RHS side of correspondence may be linked (according to Kapranov) to a study of 2-representations.

In any case, the resulting structure is basically different from the local geometric Drinfeld correspondence, which includes connections on line bundles, $D$-modules and the Fourier-Mukai transformation (see survey [F1]). The fundamental point of this difference is the fact that the proposed construction takes into account the arithmetic nature of the ground field $\mathbb{C}$ which is manifested in that it may be a completion of a global number field in some infinite point. When a curve over $\mathbb{C}$ is an archimedean fiber of an arithmetic surface $X$, the field $\mathbb{C}$ can be identified with the local completions of the 
number fields related to all horizontal curves on $Y$. The picture in [P2][section $4]$ is a simplest example. There, on the arithmetical surface $X=\mathbb{P}^{1} / \operatorname{Spec}(\mathbb{Z})$ we see three horizontal curves defined by the equations $t=0 ; t=1 / 5 ; t=2$ where $t$ is a coordinate on the general fiber $\mathbb{P}^{1} / \mathbb{R}$ which is the curve lying over the infinite place $(\infty)$ of the ground "curve" $=$ "compactification"of $\operatorname{Spec}(\mathbb{Z}))$.

At the same time, the Drinfeld construction makes sense over any ground field (of characteristic 0 ?) and does not depend on its nature.

Remark 3. Also, there should be a similar picture for curves over $\mathbb{R}$. There arise the local fields of the form $\mathbb{R}((t))$ and for them we have

$$
\begin{aligned}
& K_{2} \mathbb{R}[[t]] \hookrightarrow K_{2} \mathbb{R}((t)) \rightarrow K_{1} \mathbb{R}=\mathbb{R}^{*} \\
& K_{2} \mathbb{R}[[t]] \rightarrow K_{2} \mathbb{R} \rightarrow\{ \pm 1\}
\end{aligned}
$$

These expansions correspond to unramified extension $\mathbb{C}((t)) \supset \mathbb{R}((t))$ and to tamely ramified extension $\mathbb{R}\left(\left(t^{1 / 2}\right)\right) \supset \mathbb{R}((t))$. There are no other abelian extensions of the field $\mathbb{R}((t))$. The class field theory for the field $K=\mathbb{R}((t))$ is a canonical isomorphism

$$
K_{2}(K) /\{\text { divisible elements }\} \rightarrow \operatorname{Gal}\left(K^{a b} / K\right) .
$$

As above, we assume that the Weil group of the field $\mathbb{R}((t))$ can be constructed from the group $\left.K_{2} \mathbb{R}((t))\right)$.

Consequently, the basic fields of the Langlands program should be supplemented by finite extensions of the fields $\mathbb{R}(t)$ and $\mathbb{Q}_{p}(t)$, associated with algebraic curves defined over fields $\mathbb{R}$ and $\mathbb{Q}_{p}$. To the list of local fields, we have to add fields $\mathbb{R}((t))$ and $K((t))$ with $K \supset \mathbb{Q}_{p}$.

Remark 4. In the Langlands correspondence, a fundamental role played by $L$-functions. One can attach to the representations of Galois groups (and more general of the Weil groups) certain $L$-functions (in particular, these are the Dedekind and Hecke $L$-functions for abelian representations and their generalization to the non-abelian case, introduced by E. Artin). On the other hand, R. Langlands (and then R. Godement and H. Jacquet) introduced the $L$-functions for automorphic representations of adelic groups of reductive groups. As the arithmetic $L$-functions, they determined by an Euler product of local $L$-factors, associated with the local components of a global automorphic representation (see the section on Hasse-Weil conjecture).

An important property of the Langlands correspondence is the equality of $L$-functions which stand in both parts of the correspondence, arithmetic $L$ functions and automorphic $L$-functions. Global equality precedes by equalities of local $L$-functions for all the local correspondences. 
In the case of geometric Langlands correspondence, introduced by Drinfeld, apparently there is no proper definition of $L$-functions and hence there is no any equality of this kind. Our interpretation of geometric correspondence suggests that there have to exist $L$-functions associated with the local fields $\mathbb{C}\left(\left(t_{P}\right)\right)$ of points $P$ on the curve $X$ defined over $\mathbb{C}$. They should be included in the local geometric Langlands correspondence. Since there is a structure map $X \rightarrow \operatorname{Spec}(\mathbb{C})$, one needs to take into account that the field $\mathbb{C}$ has its own $L$-function entered into the local Langlands correspondence for this field. They are the gamma functions, associated with Hodge structures according to Serre [S3].

Since we expect that there are certain direct and inverse images of automorphic forms associated with the map $f: X \rightarrow \operatorname{Spec}(\mathbb{C})$, this suggests that the hypothetical $L$-functions of local fields $\mathbb{C}\left(\left(t_{P}\right)\right)$ for the points on $X$ must be associated with these gamma functions. Let $z \in \mathbb{C}^{*}$ and $[z]=z /|z|,|z|_{\mathbb{C}}=$ $|z|^{2}$. Then the characters of $\mathbb{C}^{*}$ have the form

$$
\chi: z \mapsto[z]^{l}|z|_{\mathbb{C}}^{t} \quad l \in \mathbb{Z} \quad t \in \mathbb{C} .
$$

As a preliminary definition of the local $L$-function corresponding to unramified extensions at the point $P$ of $X$, one can offer the same gamma-factors for the character $\chi$ as in the one-dimensional situation of the local field $\mathbb{C}[\mathbf{K n}]$ :

$$
L_{P}(s, \chi)=2(2 \pi)^{-(s+t+|l| / 2)} \Gamma(s+t+|l| / 2) .
$$

Here we assume that the unramified "extensions" of the field $K_{P}=\mathbb{C}\left(\left(t_{P}\right)\right)$ can be described by the Weil group of its residue field $\mathbb{C}$. As we noted above, although the field $\mathbb{C}$ has no nontrivial extensions, the role of the Galois group played by its Weil group when we build the local Langlands correspondence.

Next, we can assume that global $L$-function of a sheaf $\mathcal{F}$ on $X$ will decompose into a product over all points of the curve of these local $L$ functions.

\section{Direct image conjecture}

Let $f: X \rightarrow B$ be a proper morphism of a surface $X$ onto a regular curve $B$ with a smooth general fiber. Return back to the hypothesis expressed above about the existence of direct image from the set of abelian characters of $K_{2}\left(\mathbb{A}_{X}\right)$ to the set of automorphic representations of $\mathrm{GL}\left(\mathbb{A}_{B}\right)$ on $B$. Let us formulate a number of properties which must be satisfied. The our starting point are well-known properties of the direct image (= automorphic induction) in one-dimensional case. Here they are (see, for example, $[\mathbf{A C}],[\mathbf{H H}][$ Prop. $4.5])$. 
So, let $f: B^{\prime} \rightarrow B$ be a finite unramified covering of $B^{\prime}$ and $B$ of degree $m$. Then the direct image $f_{*}$ (= automorphic induction $\left.A I\right)$ is a mapping $A I: \mathcal{A F}^{n r}\left(n, B^{\prime}\right) \rightarrow \mathcal{A F}^{n r}(n m, B)$, and the inverse image $f^{*}$ (= base change $B C)$ is a mapping $B C: \mathcal{A F}^{n r}(n, B) \rightarrow \mathcal{A F}^{n r}\left(n, B^{\prime}\right)$. Here, $\mathcal{A F}^{n r}(n, B)$ is the space of all unramified automorphic forms on $\operatorname{GL}\left(n, \mathbb{A}_{B}\right)$ :

$$
\mathcal{A F}^{n r}(n, B)=\left\{\text { smooth functions on } \operatorname{GL}(n, \mathcal{O}) \backslash \mathrm{GL}\left(n, \mathbb{A}_{B}\right) / \mathrm{GL}(n, K)\right\} \text {, }
$$

where $\mathcal{O}=\prod_{x} \mathcal{O}_{x}, x \in B$ and $K=\mathbb{F}_{q}(B)$. We have the following properties:

i) if $\phi \in \mathcal{A F}^{n r}\left(n, B^{\prime}\right)$ and $\psi \in \mathcal{A} \mathcal{F}^{n r}(k, B)$, then the projection formula holds

$$
f_{*}\left(\phi \bigotimes f^{*}(\psi)\right)=f_{*}(\phi) \otimes \psi^{18}
$$

ii) $f_{*}(\check{\phi})=\check{f}_{*}(\phi) \quad f_{*}(\check{\phi})=\check{f}_{*}(\phi)$, where $\check{\phi}(g)=\phi\left({ }^{t} g^{-1}\right)$ (duality formula);

iii) if $n=1$, then $f_{*}(\chi)(g z)=\omega(z) f_{*}(\chi)(g)$ and $f_{*}(1)(g z)=\omega_{0}(z) f_{*}(1)(g)$ where $z \in \mathbb{A}_{B}^{*}=$ center of the group $\operatorname{GL}\left(\mathbb{A}_{B}\right)$ and $\omega \omega_{0}^{-1}=\beta^{*}(\chi)$ for the natural embedding $\beta: \mathbb{A}_{B}^{*} \rightarrow \mathbb{A}_{B^{\prime}}^{*}$;

iv) for unramified coverings $f^{\prime}: B^{\prime \prime} \rightarrow B^{\prime}$ and $f: B^{\prime} \rightarrow B$, we obtain

$$
\left(f^{\prime} \circ f\right)^{*}=f^{*} \circ f^{*} \quad\left(f^{\prime} \circ f\right)_{*}=f_{*} \circ f_{*}^{\prime} ;
$$

$\mathbf{v})$ if $k: B^{\prime} \rightarrow B^{\prime}$ and $j: B \rightarrow B$ are automorphisms of curves for which $f \circ k=j \circ f$, then the following base change rules hold ${ }^{19}$

$$
k^{*} \circ f_{*}=j^{*} \circ f_{*} \quad \text { and } \quad k_{*} \circ f^{*}=j_{*} \circ f^{*}
$$

vi) if $\phi \in \mathcal{A F}^{n r}\left(n, B^{\prime}\right)$ then $L_{\mathrm{GL}\left(n, \mathbb{A}_{B^{\prime}}\right)}(\phi)=L_{\mathrm{GL}\left(n m, \mathbb{A}_{B}\right)}\left(f_{*}(\phi)\right)$.

For the nonramified case, the definition of $L$-functions is given below on the section on the Hasse-Weil conjecture. These properties are supposed to be true for much more general case of ramified coverings. As far as I know, they were established, in the number fields case, only for the Galois cyclic extensions $[\mathbf{A C}]$. In the geometric case, the existence of automorphic induction with these properties follows from the Lafforgue's theorem (see footnote 21) and the corresponding construction for the Galois groups.

\footnotetext{
${ }^{18}$ The operation $\otimes$ creates from the forms on the groups $\operatorname{GL}\left(n, \mathbb{A}_{B}\right)$ and $\operatorname{GL}\left(n^{\prime}, \mathbb{A}_{B}\right)$ a form on the group $\mathrm{GL}\left(n, \mathbb{A}_{B}\right) \times \mathrm{GL}\left(n^{\prime}, \mathbb{A}_{B}\right)$ which corresponds to the external tensor product of representations.

${ }^{19}$ Let us make a terminological remark. Here, the expression "base change"means as it is customary in algebraic geometry. The same expression was understood above as it is usually done in the theory of automorphic representations.
} 
We show how to obtain the property iii) from the corresponding property for the direct images of $l$-adic sheaves. We have the following equality wellknown in the theory of representations:

$$
\operatorname{Det} \rho(g) \operatorname{Det} r(g)^{-1}=\chi(\operatorname{Ver}(g))
$$

for representations $\rho$ and $r$ of a group $G$. Here, $H \subset G$ is a subgroup of finite index, $\chi: H \rightarrow \mathbb{C}^{*}$ is a character, $\rho=\operatorname{ind}_{H}^{G}(\chi), r=\operatorname{ind}_{H}^{G}(1), g \in G$ and Ver $: G^{a b} \rightarrow H^{a b}$ is the transfer (Verlagerung) (definition of the latter, see $[\mathrm{AT}][$ ch. XIII.2]).

For a finite unramified covering $f: B^{\prime} \rightarrow B$, we have an embedding of the fundamental group $\pi_{1}(B) \rightarrow \pi_{1}\left(B^{\prime}\right)$ to a subgroup of finite index. Applying (8) to their representations we get the following formula for the determinant of the image of a locally constant $l$-adic sheaf $\mathcal{F}$ of rank 1 on the curve $B^{\prime}$

$$
\operatorname{Det} f_{*} \mathcal{F} \otimes\left(\operatorname{Det} f_{*} \mathbb{Q}_{l}\right)^{-1}=\mathrm{Nm}_{B^{\prime} / B} \mathcal{F},
$$

where $\operatorname{Det}(\mathcal{G})_{b}=\operatorname{Det}\left(\mathcal{G}_{b}\right), b \in B$ and the norm of the sheaf is defined as

$$
\left(\mathrm{Nm}_{B^{\prime} / B} \mathcal{F}\right)_{b}=\otimes_{b^{\prime} \mapsto b} \mathrm{Nm}_{k\left(b^{\prime}\right) / k(b)} \mathcal{F}_{b^{\prime}}
$$

To get from this the property iii) one needs to apply the commutative diagram arising from the class field theory,

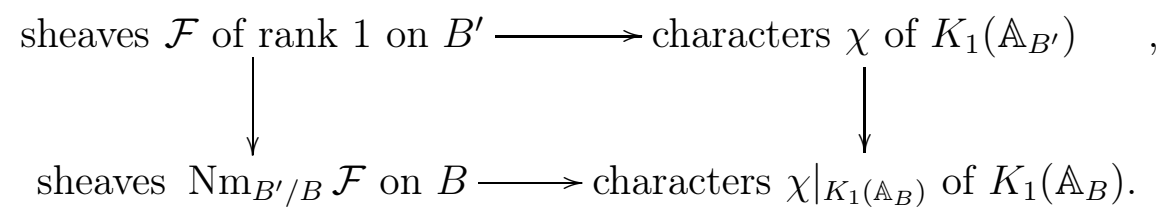

where the horizontal arrows are abelian Langlands correspondences, and to build the right vertical arrow we made use the natural embedding of $K_{1}\left(\mathbb{A}_{B}\right) \rightarrow K_{1}\left(\mathbb{A}_{B^{\prime}}\right)$. Then one applies the formula for the central character of automorphic representations of the adele group that corresponds to the $l$ adic sheaf according to the Langlands correspondence (Lafforgue's theorem). That is, the central character is the image of the sheaf Det $f_{*} \mathcal{F}$ of rank 1 with respect to the abelian Langlands correspondence.

When we turn to two-dimensional case, the number of functorial properties will be increasing and the properties itself are more complicated. Recall that we consider maps $f_{*}^{i}, i=0,1,2$ from the group $\mathcal{A F}^{n r}\left(K_{2}\left(\mathbb{A}_{X}\right)\right)$ of unramified characters of $K_{2}\left(\mathbb{A}_{X}\right)$, satisfying the reciprocity law (2) (and trivial on subgroup $K_{2}\left(\mathbb{A}_{12, X}\right)$ ), to the space of unramified automorphic forms $\mathcal{A F}^{n r}\left(\mathrm{GL}\left(r_{i}, \mathbb{A}_{B}\right)\right.$. Note that the ranks $r_{i}$ depend, in general, on $\chi$. We denote by $g$ the genus of a general fiber of the map $f$. 
Direct image conjecture. For smooth proper morphisms $f: X \rightarrow B$ of a smooth surface $X$ onto a smooth proper curve $B$, defined over a finite field $\mathbb{F}_{q}$, there exist homomorphisms $f_{*}^{i}: \mathcal{A F}^{n r}\left(K_{2}\left(\mathbb{A}_{X}\right)\right) \rightarrow \mathcal{A F}^{n r}\left(\operatorname{GL}\left(r_{i}, \mathbb{A}_{B}\right)\right.$ $(i=0,1,2)$ such that

o) for fiber-trivial $\chi, r_{0}=r_{2}=1$ and $r_{1}=2 g$. For non-trivial $\chi, r_{0}=r_{2}=0$ and $r_{1}=2 g-2$;

i) if $\chi \in \mathcal{A F}^{n r}\left(K_{2}\left(\mathbb{A}_{X}\right)\right)$ and $\eta \in \mathcal{A} \mathcal{F}^{n r}\left(K_{1}\left(\mathbb{A}_{B}\right)\right)$, then the projection formula has the form

$$
f_{*}^{i}\left(\chi \otimes f^{*}(\eta)\right)=f_{*}^{i}(\chi) \otimes(\eta \circ \operatorname{det}),
$$

where det $: \operatorname{GL}\left(r_{i}, \mathbb{A}_{B}\right) \rightarrow \mathbb{A}_{B}^{*}$ is the determinant;

ii) for the direct images of a character $\chi \in \mathcal{A F}^{n r}\left(K_{2}\left(\mathbb{A}_{X}\right)\right)$, the duality formula holds

$$
\check{f}_{*}^{i}(\chi)=f_{*}^{2-i}\left(\chi^{-1} \otimes|\cdot|\right),
$$

where $|\cdot|$ is the canonical character (norm) on the group $K_{2}\left(\mathbb{A}_{X}\right)$ (see $[\mathbf{P 1}])$;

iii) $f_{*}^{i}(\chi)(g z)=\omega_{i}(z) f_{*}^{i}(\chi)(g)$ and $f_{*}^{i}(1)(g z)=\omega_{i}^{0}(z) f_{*}^{i}(1)(g)$ where $z \in$ $\mathbb{A}_{B}^{*}=$ center of the group $\operatorname{GL}\left(\mathbb{A}_{B}\right)$ and

$$
\prod_{i} \omega_{i}^{(-1)^{i}}(z)\left(\prod_{i} \omega_{i}^{0(-1)^{i}}(z)\right)^{-1}=\chi\left(\left(\omega_{X / B}\right), \beta^{*}(z)\right)
$$

where $\beta: \mathbb{A}_{B}^{*} \rightarrow\left(\mathbb{A}_{X}^{02}\right)^{*}$ is the natural embedding, $\left(\omega_{X / B}\right) \in\left(\mathbb{A}_{X}^{01}\right)^{*}$ is an idele corresponding to the relative canonical class (see $[\mathbf{P} \mathbf{1}]$ ) and $(-,-): \mathbb{A}_{X}^{*} \times \mathbb{A}_{X}^{*} \rightarrow K_{2}\left(\mathbb{A}_{X}\right)$ denotes multiplication in the $K$-theory;

iv) for a finite unramified covering $g: B \rightarrow B^{\prime}$ of degree $m$ we have

$$
(f \circ g)_{*}^{i}(\chi)=g_{*} \circ f_{*}^{i}(\chi)
$$

on the group $\operatorname{GL}\left(r_{i} m, \mathbb{A}_{B^{\prime}}\right)^{20}$;

v) if $k: X \rightarrow X$ and $j: B \rightarrow B$ are automorphisms of schemes for which $f \circ k=j \circ f$, then the following formula for base change is true

$$
k^{*} \circ f_{*}^{i}=j^{*} \circ f_{*}^{i} \quad \text { and } \quad k_{*} \circ f^{*}=j_{*} \circ f^{*}
$$

\footnotetext{
${ }^{20} \mathrm{~A}$ composition rule for finite unramified coverings of the scheme $X$ must also be the case, but it involves going beyond the abelian group $K_{2}\left(\mathbb{A}_{X}\right)$.
} 


$$
L_{X}(\chi)=\prod_{i} L_{\mathrm{GL}\left(r_{i}, \mathbb{A}_{B}\right)}\left(f_{*}^{i}(\chi)\right)^{(-1)^{i}} ;
$$

vii) there must also be a simple formula for the behavior under blowings of the scheme $X$ (the reader, tinker!).

In fact, this hypothesis can be proved. Namely, we have

Theorem. The existence of maps $f_{*}^{i}$, satisfying the properties 0) - vi) for smooth proper morphisms $f: X \rightarrow B$, follows from the existence of the global Langlands correspondence for algebraic curve $B$ over finite fields.

We prefer to consider this statement as a hypothesis, as there is a very non-trivial task to get an explicit construction of the direct image, without using the Langlands correspondence and the general theory of etale cohomology. We now give an outline of how to get this construction, using Langlands correspondence and its properties. Referring to the diagram (5), let us define the mappings $f_{*}^{i}(\chi)$, using the two horizontal maps in this diagram and the left vertical map. Upper arrow, the two-dimensional abelian Langlands correspondence is a bijection, which allows to invert it and thus to begin the construction of the direct image. Left arrow follows from the theory of étale $l$-adic sheaves. Finally, the most non-trivial part of the construction is the bottom arrow, the global Langlands correspondence for $B$ (Lafforgue's theorem $[\mathbf{L}]^{21}$ ).

We sketch proofs of the most non-trivial properties of the direct image.

For the property o), we say that the character $\chi$ is fiber-trivial if its restriction on a fiber $F_{b}$ is trivial on the group $\operatorname{Pic}^{0}\left(F_{b}\right)$ for some $b \in B$. The property follows easily from the known facts on the cohomology of l-adic sheaves.

Since $X$ is a surface over a finite field, the duality formula ii) follows from the relative duality theorem for $l$-adic sheaf $\mathcal{F}$ on the scheme $X$, corresponding to the character $\chi$ :

$$
\begin{aligned}
& R^{i} f_{*}(\mathcal{F}) \otimes R^{2-i} f_{*}\left(\operatorname{Hom}\left(\mathcal{F}, \mathbb{Q}_{l}\right)(1)\right) \rightarrow R^{2} f_{*}\left(\mathcal{F} \otimes \operatorname{Hom}\left(\mathcal{F}, \mathbb{Q}_{l}\right)(1)\right) \\
& \left.\rightarrow R^{2} f_{*}\left(\mathbb{Q}_{l}\right)(1)\right)=\mathbb{Q}_{l}
\end{aligned}
$$

and subsequent application of the Langlands correspondence.

\footnotetext{
${ }^{21}$ In the Lafforgue's work the correspondence was constructed for irreducible representations of the Galois group and cuspidal representations of adele group on the curve. Apparently, the parabolic induction technique allows us to reduce the general case of completely irreducible representations to this one. This follows from Yu. G. Zarhin's semi-simplicity theorem for the Tate module of abelian varieties.
} 
In order to obtain the property iii), it is necessary to formulate an analogue of (9) in the new situation. We set

$$
\left(\mathrm{Nm}_{X / B} \mathcal{F}\right)_{b}=\otimes_{x \in f^{-1}(b)} \mathcal{F}_{x}^{\nu_{x}\left(\omega_{X / B}\right)}
$$

where $\mathcal{F}$ is a sheaf of rank 1 on $X, b \in B, \omega_{X / B}$ is a section of the relative cotangent bundle and $\nu_{x}$ is the valuation at the point $x$ on a fiber of the mapping $f$. We then have

$$
\otimes_{i}\left(\operatorname{Det} R^{i} f_{*} \mathcal{F}\right)^{(-1)^{i}} \otimes_{i}\left(\operatorname{Det} R^{i} f_{*} \mathbb{Q}_{l}\right)^{(-1)^{i+1}}=\mathrm{Nm}_{X / B} \mathcal{F} .
$$

This relation follows when we apply to the fibers of the morphism $f$ Deligne's formula for a locally constant sheaf $\mathcal{F}$ of rank 1 on a smooth projective geometrically irreducible curve $C$ of genus $g$ and defined over a field $k$. It reads as

$$
\otimes_{i}\left(\operatorname{Det} H^{i}(C, \mathcal{F})^{(-1)^{i}}=\otimes_{x \in C} \mathcal{F}_{x}^{\nu_{x}(\omega)}(1-g),\right.
$$

where $\omega$ is a non-zero differential form on $C$ of degree 1 and $\mathcal{F}(n)$ is the Tate's twist of the sheaf $\mathcal{F}$ (see, e.g, $[\mathbf{P} 1][n \text { 3.1 }]^{22}$ ).

Morphism $f$ gives the inverse map

$$
i: K_{1}\left(\mathbb{A}_{B}\right) \rightarrow K_{2}\left(\mathbb{A}_{X}\right), \quad i(a)=\left(a,\left(\omega_{X / B}\right)_{02}\right), a \in K_{1}\left(\mathbb{A}_{B}\right)
$$

where $\left(\omega_{X / B}\right)_{02} K_{1}\left(\mathbb{A}_{X, 02}\right)$ is the idele associated with the section $\omega_{X / B}$ (see [P1][n 2.2]). We can now formulate the analogue of diagram (10)

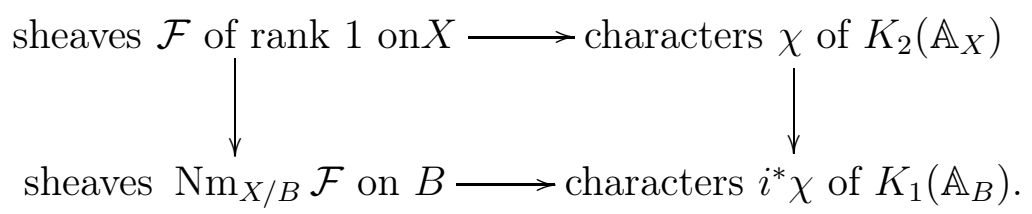

Then the same arguments as in the case of a finite covering, give the property iii).

To understand the property vi), the group $K_{2}\left(\mathbb{A}_{X}\right)$ can be presented as an adelic product over all flags $x \in C$ and then computed in the following way

$$
K_{2}\left(\mathbb{A}_{X}\right) \rightarrow K_{2}\left(\mathbb{A}_{X}\right) / K_{2}\left(\mathbb{A}_{12}\right)=\oplus_{x \in C} \mathbb{Z}=\oplus_{x \in X} \oplus_{C \ni x} \mathbb{Z} \rightarrow \oplus_{x \in X} \mathbb{Z} \cdot 1_{x}
$$

\footnotetext{
${ }^{22}$ If $k=\mathbb{F}_{q}$ and the sheaf $\mathcal{F}$ corresponds to a character $\chi$ of the idele group $K_{1}\left(\mathbb{A}_{C}\right)$, then (11) is a reformulation of the expression for the elementary factor of the classical functional equation $L_{C}(s, \chi)=\chi((\omega)) q^{s(2-2 g)} q^{g-1} L_{C}\left(1-s, \chi^{-1}\right)$ for $L$-functions $L_{C}(s, \chi)$ of the curve $C$. The elementary factor $\chi((\omega)) q^{s(2-2 g)} q^{g-1}$ corresponds to an action of the Frobenius automorphism of the ground field on the one-dimensional $l$-adic space dual to the space from the LHS in $(11)$. The factor $\chi((\omega)) q^{s(2-2 g)}$ is associated with the product $\otimes_{x \in C} \mathcal{F}_{x}^{\nu_{x}(\omega)}$, and the twisting $(1-g)$ corresponds to $q^{g-1}$.
} 
Here, the last arrow is the sum of all $x, C$-components around the given point $x$. Finally, since the character $\chi$ is unramified and automorphic the reciprocity law implies that $\chi$ is defined on the latter group ${ }^{23}$.

Then,

$$
L_{X}(\chi):=\prod_{x \in|X|}\left(1-\chi\left(1_{x}\right)\right)^{-1},
$$

where $1_{x}$ is a generator of the local group at the point $x \in X$. The $L$ functions $L_{\mathrm{GL}\left(r_{i}, \mathbb{A}_{B}\right)}\left(f_{*}^{i}(\chi)\right)$ are the standard $L$-functions attached to the representations generated by the forms $\left.f_{*}^{i}(\chi)\right)$ (see $[\mathbf{G J}]$ and the next section).

This property follows from the analogous property

$$
L_{X}(\mathcal{F})=\prod_{i} L_{B}\left(R^{i} f_{*}(\mathcal{F})\right)^{(-1)^{i}}
$$

for the corresponding $l$-adic sheaves $\mathcal{F}$ and $R^{i} f_{*}(\mathcal{F})$ and the Langlands correspondences on $X$ and $B$ which imply that $L_{X}(\mathcal{F})=L_{X}(\chi)$ and $L_{B}\left(R^{i} f_{*}(\mathcal{F})\right)=$ $L_{\mathrm{GL}\left(r_{i}, \mathbb{A}_{B}\right)}\left(f_{*}^{i}(\chi)\right)$ for all $i$.

Remark 5. It is not difficult to state local and semi-local versions of this conjecture (as we deal above) and formulate their interrelations. We can even give an explicit expression for these direct images in the case of a (smooth) mapping $f: X_{b} \rightarrow \operatorname{Spec} \mathcal{O}_{b}$ with general fiber of genus $g$, and the character $\chi$ is trivial (or is the $s$-th degree of the norm $|\cdot|$ ). Then, $f_{*}^{0}(\chi)=|\cdot|^{s}, f_{*}^{1}(\chi)=$ $|\cdot|^{s-1}$, and $f_{*}^{1}(\chi)$ is an Eisenstein series generating an unramified principal series representation of group $\mathrm{GL}\left(2 g, K_{b}\right)$. The Satake parameters of this representation are eigenvalues of the Frobenius automorphism of the field $k(b)$, acting on 1-dimensional cohomology of the curve $F_{b}=X_{b} \times_{\hat{\mathcal{O}}_{b}} k(b)$. Still, we can define them without any application of the $l$-adic cohomology making use of the Tate-Iwasawa method on the curve $F_{b}$. The appropriate version of this method was given in $[\mathbf{P} 4]$.

Remark 6. Certainly, the choice of smooth morphisms in our conjecture is too restrictive (since we have a complete base $B$ ). There would be more reasonable to consider the semi-stable families $X$ over a proper curve $B$. In this case, we can expect that the target of the direct image (still for unramified $\chi$ on $X$ ) will be tamely ramified automorphic representations $\left(\pi, V_{\pi}=\otimes_{b \in B} V_{\pi, b}\right)$ of the groups $\operatorname{GL}\left(\mathbb{A}_{B}\right)$. If $\operatorname{dim}_{\mathbb{C}} V_{\pi, b}^{I}=1$ for the Iwahori subgroups $I \subset \operatorname{GL}\left(\mathcal{O}_{b}\right)$ and all the points $b$ of bad reduction then we attach to $\pi$ a tamely ramified automorphic function on $\operatorname{GL}\left(\mathbb{A}_{B}\right)$. Since for general

\footnotetext{
${ }^{23}$ and actually on its quotient $K_{2}\left(\mathbb{A}_{X}\right) / K_{2}\left(\mathbb{A}_{12}\right) K_{2}\left(\mathbb{A}_{01}\right) K_{2}\left(\mathbb{A}_{02}\right)$ which is isomorphic to the second Chow group. This group is an extension of $\mathbb{Z}$ by a finite group according to the S. Bloch and K. Kato finiteness theorems.
} 
tamely ramified representations $V_{\pi}$ of a group $\operatorname{GL}\left(K_{b}\right), \operatorname{dim}_{\mathbb{C}} V_{\pi}^{I}>1$, to construct such function we need additional reasons. This is possible for the semi-stable elliptic families $[\mathbf{L} \mathbf{2}, \mathbf{G}]$ where the special representations are the local components for the points of bad reduction.

Remark 7. In the geometric case, the direct image conjecture follows from the two-dimensional class field theory and the Langlands correpondence on the curve $B$ (Lafforgue's theorem). In general, if it were possible to determine not only the abelian automorphic representations, but also automorphic representations of the non-commutative groups of adeles on schemes $X$ of any dimension (by developing, for example, Kapranov's idea), then one could try to define the operation of the direct image $f_{*}^{i}$, expanding the map $f$ in a composition of the projection of a projective bundle and of an embedding. This is suggested by the Grothendieck's proof of the Riemann-Roch theorem.

\section{A link with the Hasse-Weil conjecture}

We assume that the conjecture on the direct image can be stated and proved in much more general situation. Namely, one can remove the smoothness condition for schemes and morphisms (see Remark 6) and thus consider the ramified characters. Finally, there is an extension of the conjecture to the number field case. Then, an (explicit) construction of direct images of this type would provide an opportunity to prove the Hasse-Weil conjecture [W2] on meromorphic continuation and functional equation for $L$-functions of algebraic curves defined over algebraic number fields (and reprove the corresponding Grothendieck theorem on $L$-functions of algebraic curves defined over fields of algebraic functions with a finite field of constants, without any use of $l$-adic cohomology).

Curves with this field of definition have models, two-dimensional schemes $X$ with a structure morphism on a one-dimensional scheme $B$, either a curve over a finite field or a spectrum of a ring of integers in an algebraic number field. The existence of the direct image from $X$ to $B$ allows to build an automorphic form on $B$, starting from a character of the group $K_{2}\left(\mathbb{A}_{X}\right)$. The Mellin transformation of this form will be the $L$-function of interest to us. Automorphy of the constructed in this way form shall, in accordance with the general theory, imply meromorphic continuation and functional equation of the $L$-function [W3, JL, GJ].

We give a precise formulation of the corresponding theorem for the case of algebraic curves defined over a finite field. Let $\mathcal{A} \mathcal{F}(n, B)$ be the space of automorphic forms on $\operatorname{GL}\left(n, \mathbb{A}_{B}\right)$, i.e. of the functions $F$ on $\operatorname{GL}\left(n, \mathbb{A}_{B}\right)$ such that: 
i) there exists a character $\omega$ of the center $Z=\mathbb{A}_{B}^{*}$ of $\mathrm{GL}(n)$ such that $F(z g)=\omega(z) F(g), z \in Z, g \in \mathrm{GL}\left(n, \mathbb{A}_{B}\right)$;

ii) if $\gamma \in \mathrm{GL}(n, K)$, where $K=\mathbb{F}_{q}(B)$, then $F(g \gamma)=F(g)$;

iii) there exists an open compact subgroup $\mathbb{K}^{\prime} \subset \mathrm{GL}(n, \mathcal{O})$, where $\mathcal{O}=$ $\prod_{x} \mathcal{O}_{x}, x \in B$, such that $F(k g)=F(g), k \in \mathbb{K}^{\prime}, g \in \mathrm{GL}\left(n, \mathbb{A}_{B}\right)$.

The group $\operatorname{GL}\left(n, \mathbb{A}_{B}\right)$ acts on the space of automorphic forms by left translations. Smooth representation of GL $\left(n, \mathbb{A}_{B}\right)$ is called em automorphic if it is embedded as a sub-representation in the space of automorphic forms $\mathcal{A F}(n, B)$.

Each irreducible automorphic representation $\pi$ is a tensor product $\otimes_{x \in B} \pi_{x}$ of smooth irreducible representations of local groups $\mathrm{GL}\left(n, K_{x}\right)$. For almost all $x$ the representation $\pi_{x}$ is a spherical one [Fl], [B] [Chap. 3.4]. According to the general theory, such a representation belongs to the principal series, i.e. can be obtained by parabolic induction from an unramified character $\chi: T \rightarrow \mathbb{C}^{*}$ of a maximal torus $T$ of the group $\mathrm{GL}\left(n, K_{x}\right)$ (see [B] [theorem 4.6.4] for the case $n=2$, the proof of which can be extended to an arbitrary $n$ ). This character is given by a set of $n$ complex numbers $z_{1}, \ldots, z_{n}$ (defined up to a permutation). Then we can define the local $L$-function for spherical representations of $\pi_{x}$ as

$$
L_{B}\left(s, \pi_{x}\right)=\prod_{i}\left(1-z_{i} q^{-s}\right)^{-1}, i=1, \ldots, n .
$$

and the global $L$-function as the Euler product

$$
\left.L_{B}(s, \pi)=\prod_{x} L_{(} s, \pi_{x}\right) .
$$

Missing factors for a finite set of points $x \in B$ are defined in a more complicated way (see [JL] [Ch. 1, Theorem 2.18], [G] [Ch. 2, Theorem 8.2 and Table A] for $n=2$ and [GJ] [Ch. 1, Theorem 3.3] for an arbitrary $n$ ).

Theorem. Let $B$ be a smooth projective curve over a finite field $\mathbb{F}_{q}$ and let $\pi$ be an automorphic representation of $\mathrm{GL}\left(n, \mathbb{A}_{B}\right)$. Then:

i) the Euler product (12) for the $L$-function $L_{B}(s, \pi)$ converges in the right half-plane $\operatorname{Re}(s)>1$;

ii) $L_{B}(s, \pi)$ extends meromorphically to the whole s-plane as a rational function of $q^{-s}$;

iii) $L_{B}(s, \pi)=\epsilon(s, \pi) L_{B}(1-s, \check{\pi})$, where $\epsilon(s, \pi)$ is an elementary factor of the form $a q^{b s}, a, b \in \mathbb{R}$. 
The theorem was proven for cuspidal representations in [GJ] [Theorem 8.13]. The general case reduces to this one by using parabolic induction $[\mathbf{J}]$ [Theorem 6.2]. For the group GL(2), the result was obtained by Jacquet and Langlands in 1970 [JL] [Ch. II, Theorem 11.1]. Note that in these works an analogue of this theorem for number fields was also proven.

If all the local components of the representation $\pi$ are spherical, then it is generated by an unramified automorphic form (=tensor product of the local spherical vectors) and its Mellin transform is equal to the $L$-function $L_{B}(s, \pi)$. This fact allows us to apply this theorem to automorphic forms $f_{*}^{i}(\chi)$, arising as direct images of an unramified automorphic character $\chi$ of $K_{2}\left(\mathbb{A}_{X}\right)$ on a surface $X$, which are mapped onto the curve $B$.

Such a construction can be seen as a way of solving the general problem, discussed in [P3][5.2]. It is a generalization of the Tate-Iwasawa approach $[\mathbf{T} \mathbf{1}, \mathbf{I w}]$ to the zeta- and $L$-functions of one-dimensional schemes to the schemes of higher dimension (in this case of dimension 2). It seems that the harmonic analysis on two-dimensional adelic spaces [P3][2] and the theory of representations of discrete Heisenberg groups [P3][3, 4] are the necessary tools to build this (hypothetical) direct image.

Remark 8. Let $X$ be an $n$-dimensional regular scheme and let $f: X \rightarrow B$ be a plat proper morphism onto a one-dimensional regular scheme $B$ with smooth general fiber. In the number field case, the schemes $X$ and $B$ must be considered in the Arakelov geometry, completed by archimedean fibers . The $n$-dimensional class field theory suggests that there exists a reciprocity map

$$
\varphi_{X}: K_{n}^{M}\left(\mathbb{A}_{X}\right) \longrightarrow G_{K}^{a b},
$$

where $K_{n}^{M}\left(\mathbb{A}_{X}\right)$ is the Milnor part of the $n$-th Quillen's K-functor and $G_{K}^{a b}$ is the Galois group of the maximal abelian covering of the scheme $X$. We may assume that the direct image conjecture is valid (with the appropriate changes) also for these morphisms and automorphic characters of the group $K_{n}^{M}\left(\mathbb{A}_{X}\right)$. Automorphy of the characters means that they are trivial on $n$ subgroups

$$
K_{n}^{M}\left(\mathbb{A}_{0, \hat{1}, 2, \ldots, n}\right), \quad K_{n}^{M}\left(\mathbb{A}_{0,1, \hat{2}, \ldots, n}\right), \ldots, \quad K_{n}^{M}\left(\mathbb{A}_{0,1, \ldots, \hat{n}}\right),
$$

where $\hat{i}$ means that the $i$-th index must be deleted. These relations are a generalization of the formulas (2). Just as above, this conjecture has to imply the Hasse-Weil conjecture for the scheme $X$.

The first step to get the conjecture is to construct direct images $f_{*}: K_{n}\left(\mathbb{A}_{X}^{\bullet}\right) \rightarrow$ $K_{n-k}\left(\mathbb{A}_{B}^{\bullet}\right)[k]$ for proper morphisms $f: X \rightarrow Y$ of relative dimension $k$, full adelic complexes and $n-k \geq 0$. We do not consider the reasonable 
conditions for existence of these direct images. Let us only mention that for closed regular embeddings the map $f_{*}$ depends on a choice of the local equations for the $Y$ in $X$. Nevertheless, the resulting homomorphism $f_{*}$ is defined uniquely up to a homotopy.

\section{Appendix: zero-dimensional generalization of the Langlands correspondence}

Let us consider in more detail an analogue of the Langlands correspondence in zero-dimensional situation, i.e. for finite fields and their extensions. As in the case of fields of higher dimension class field theory gives us a canonical reciprocity map

$$
K_{0}(K) \longrightarrow G_{K}^{a b}=G_{K},
$$

which explicitly looks as follows:

$$
\mathbb{Z} \longrightarrow \hat{\mathbb{Z}}
$$

and maps 1 into Frobenius automorphism $\operatorname{Fr}\left(\operatorname{Fr}(x)=x^{q}\right)$ for a field $K=\mathbb{F}_{q}$. Here $K_{0}\left(\mathbb{F}_{q}\right)$ is the Grothendieck $K$-group of category of finite dimensional vector spaces, and $1 \in K_{0}\left(\mathbb{F}_{q}\right)$ corresponds to the trivial one-dimensional space.

If $K^{\prime} / K$ is a finite extension of degree $m$, then we have the commutative diagrams

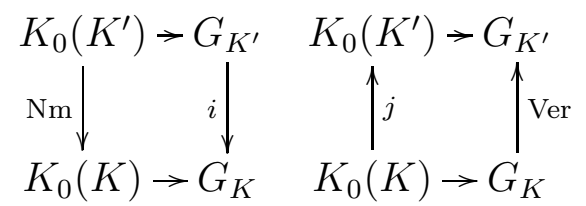

where the embeddings $i$ and $j$ induced by the inclusion of $K$ in $K^{\prime}, \mathrm{Nm}$ is the norm map and Ver is the transfer from $G_{K}^{a b}=G_{K}$ to $G_{k^{\prime}}^{a b}=G_{K^{\prime}}$. In this case, $\mathrm{Nm}$ is multiplication by $m$, and $j$ is isomorphism (of the groups equal to $\mathbb{Z})$.

As above, the map (13) leads to a homomorphism of the groups of characters (one-dimensional representations) in the opposite direction. We can define functors of direct and inverse images for the characters and these diagrams lead to their properties similar to those of the previous section.

As we remember, the Kapranov's proposal for the 0-dimensional correspondance was to take the $L$-function $L(s, \rho)$ of the Galois representation $\rho$. If $\lambda_{1}, \ldots, \lambda_{n}$ $=$ spectrum of $\rho$ then $L(s, \rho)=\operatorname{det}\left(I-\rho(F r) q^{-s}\right)=\prod_{i}\left(1-\lambda_{i} q^{-s}\right)$. On the other hand, there is a structure of $d$-representations $(d=1$ or 2$)$ for 
the correspondances in dimensions $d$. We can expect that something like 0representations might appear at the RHS of the 0-dimensional correspondance. To understand the position we give the necessary definitions of the $d$-representation theory $(d=0,1,2)$.

This is a pure algebraic theory that has nothing to do with arithmetic. We fix a group $G$, a ground field $k$ and a cardinal number $n$. The inductive structure of the theory looks then as follows:

\begin{tabular}{|c|c|c|c|c|}
\hline$d$ & $\begin{array}{c}\text { representations } \\
\text { spaces }\end{array}$ & $d$-representations & $\begin{array}{c}n=\text { dimension of } \\
\text { representation }\end{array}$ & $\begin{array}{c}\text { sets of 1-dim. } \\
\text { representations }\end{array}$ \\
\hline 2 & $\mathrm{~V}$-mod $/ k$ & $\begin{array}{c}\text { functor } \pi(g): \\
\mathrm{V} \text {-mod } \rightarrow \mathrm{V} \text {-mod }\end{array}$ & $\mathrm{rk} \mathrm{V-mod} / k$ & $H^{2}\left(G, k^{*}\right)$ \\
\hline 1 & $V / k$ & $\begin{array}{c}\text { homomorphism } \\
\pi(g): V \rightarrow V\end{array}$ & $\operatorname{dim}_{k} V$ & $\begin{array}{c}H^{1}\left(G, k^{*}\right)= \\
\operatorname{Hom}\left(G, k^{*}\right)\end{array}$ \\
\hline 0 & $A \subset \operatorname{Sym}^{n}\left(k^{*}\right)$ & just $A, \operatorname{no} \pi(g)$ & $\operatorname{deg} A=n$ & $H^{0}\left(G, k^{*}\right)=k^{*}$ \\
\hline
\end{tabular}

\begin{tabular}{|c|c|c|c|}
\hline$d$ & $\begin{array}{c}\text { distinguished } \\
\text { object }\end{array}$ & $\operatorname{Hom}^{\prime}$ s & $\begin{array}{c}\text { sets of } \\
\text { characters }\end{array}$ \\
\hline 2 & Vect/k & $\operatorname{Func}(\mathcal{C}, \mathcal{D})$ & $\left\{g \mapsto V_{g}\right\}$ \\
1 & $\mathrm{k}$ & $\operatorname{Hom}(V, U)$ & $\operatorname{Map}(G, k)$ \\
0 & 1 & $\left(\sum_{i} n_{i} a_{i}^{-1}\right)\left(\sum_{j} m_{j} b_{j}\right)$ & $k$ \\
\hline
\end{tabular}

Let here $\mathrm{V}$-mod/ $k$ be a category-module (see above), $\mathrm{rk} \mathrm{V-mod} / k$ be its rank (see $[\mathbf{G K}, \mathbf{K}]), V_{g}$ be a vector space over $k$ depending on $g \in G, V / k$ or $V$ be a vector space over $k$ and $\operatorname{Sym}^{n}\left(k^{*}\right)$ be the set of cycles of degree $n$ with non-negative multiplicities. Func $(\mathcal{C}, \mathcal{D})$ denotes the set of functors from the category $\mathcal{C}$ to the category $\mathcal{D}$ which commute with the action of the category Vect/ $k$ (in the categorical sense).

We see that for $d=0$ the group $G$ itself doesn't participate in all the constructions. For $d=0$, the direct sum of the representations $A$ and $B$ is the sum of cycles $A$ and $B$, the tensor product is their Pontryagin product in the group $k^{*}$ and dual to $A=\sum_{i} n_{i} a_{i}$ is $\check{A}=\sum_{i} n_{i} a_{i}^{-1}$. The trace $\operatorname{Tr}(A)$ is $\sum_{i} n_{i} a_{i}$ where summation is done in the group $k$. For $d=2$ the dual "object"to a category $\mathcal{C}$ is the category of functors $\operatorname{Func}(\mathcal{C} \text {, Vect } / k)^{24}$.

\footnotetext{
${ }^{24} \mathrm{I}$ 'm grateful to participants of my Steklov seminar (especially to Sergey Gorchinskiy and Denis Osipov) for a discussion that clarified some points in the final version of the table.
} 
Now, we try to apply this small theory to the local Langlands correspondence. First, the field $k=\mathbb{C}$. The group at the LHS of the correspondance is the same Galois group for all $d$. But at the RHS, we see 2-representations of the group $\mathrm{GL}(2 n, K)$ for $d=2$ and 1-representations of the group $\mathrm{GL}(n, K)$ for $d=1$. We may guess that for $d=0$ one needs to consider 0 -representations of the group $\mathrm{GL}(0, K)$, where the latter group is simply trivial. Then the Langlands correspondence could look as follows:

$\operatorname{Rep}^{(n)}\left(G_{K}\right)=\left\{n\right.$-dimensional semi-simple representations of $G_{K}$ over $\left.\mathbb{C}\right\} \stackrel{?}{\Leftrightarrow}$ $\{$ irreducible 0 -representations of the group $\mathrm{GL}(0, K)=(1)\}$,

where $\rho \in \operatorname{Rep}^{(n)}\left(G_{k}\right)$ goes to the determinant $\lambda_{1} \cdots \lambda_{n}$ of the spectrum $\lambda_{1}, \ldots, \lambda_{n}$ of $\rho$. We see that this construction does not coincide completely with the Kapranov' proposal. The $L$-function from his choice doesn't belong to the field $\mathbb{C}$ as we could expect according to the table above. Our choice is the determinant which is an element of $\mathbb{C}$. Also, it satisfies the same multiplicative property for exact sequences of representations as the $L$-function. But, the correspondence will not a bijection in contrast with the behavior of the $L$-functions which are actually the characteristic polynomials of the Frobenius automorphism. This problem we leave as a question.

Let us say that we also meet another difficulty. The Langlands correspondance is in a perfect accordance with reciprocity law for $d=1$ or 2 . However, we do not see here the group $K_{0}(K)$ at the RHS of the correspondance for $d=0$. This unexpected puzzle can be solved along the following line.

In his paper $[\mathbf{K}]$, Kapranov justifies the appearance of the non-commutative group $\mathrm{GL}(2, K)$ at the RHS of the abelian (!) 2-dimensional correspondance by the following relation

$$
K_{2}(K)=H_{2}(\mathrm{GL}(2, K), \mathbb{Z}) / H_{2}(\mathrm{GL}(1, K), \mathbb{Z})
$$

which is a particular case of the general Suslin's theorem on Milnor's $K_{n}^{M}(K)$ for a field $K$. In case of $n=2$ this could follow from the Matsumoto's theorem that $K_{2}^{M}(K)=K_{2}(K)=H_{2}(\mathrm{SL}(K), \mathbb{Z})$. Applying this, Kapranov has shown that 1-dimensional 1-representations of the $K_{2}(K)$ are in one to one correspondance with 1-dimensional 2-representations of the group $\mathrm{GL}(2, K)$ (with a trivial restriction to the subgroup $\mathrm{GL}(1, K)$ ). Since $K_{1}(K)=$ $K^{*}=H_{1}(\mathrm{GL}(1, K), \mathbb{Z})$ we have an analogous relation for 1-representations for $d=1$.

For $d=0$ we can add the relation

$$
K_{0}(K)=H_{0}(\mathrm{GL}(0, K), \mathbb{Z})=\mathbb{Z}
$$


and the 0-dimensional Langlands correspondance looks as follows

$\operatorname{Rep}^{(n)}\left(G_{K}\right)=\left\{n\right.$-dimensional semi-simple 1-representations of $G_{K}$ over $\left.\mathbb{C}\right\} \Leftrightarrow$ $\left\{n\right.$-dimensional semi-simple 1 -representations of $K_{0}(K)$ over $\left.\mathbb{C}\right\} \Rightarrow$

$\{1$-dimensional 0 -representations of the group $\mathrm{GL}(0, K)=(1)\}$.

The same versions (but with bijections) are valid also for $d=1$ or 2 . Besides that, it is unusual that we have to consider all semi-simple representations at the RHS of the correspondence but not the irreducible ones as we have for the case of dimension 1 .

\section{Список литературы}

[A] Acquista K. The Weil group of a hyper-class formation, 2005, arxiv/math 0508.078

[AT] Artin E., Tate J., Class Field Theory, Harvard, 1961.

[AC] Arthur J, Clozel L., Simple algebras, base change, and the advanced theory of the trace formula, Annals of Mathematics Studies, 120, Princeton, 1989.

[BZ] Bernstein J. N., Zelevinsky A. V. , Representations of the group GL(n, $F)$, where $F$ is a local non-archimedian field, Russian Math. Surveys, 31(1976), 1-68.

[Br] Breen L., Tannakian categories, Proc. Symp. Pure Math., Motives, Part 1, AMS, 55(1994), 337-376.

[B] Bump D., Automorphic Forms and Representations, Cambridge University Press, 1996.

[C] Cartier P., Representations of l-dic groups: a survey, Automorphic forms, representations and $L$-functions, Proc. Symposia in Pure Math., 33 (1979), Part 1, Amer. Math. Soc., Providence, 1979, 111-155.

[C] Casselman B, The L-group, Class Field Theory - Its Centenary and Prospect, Adv. Studies in Pure Math., 30(2001), 217-258.

[Dr1] Drinfeld V. G. Langlands'conjecture for GL(2) over functional fields, Proc.Intern. Congr. Math.(Helsinki,1978), Acad. Sci. Fennica, Helsinki,1980, 565-574. 
[Dr2] Drinfeld V. G. Two-dimensional l-adic representations of the fundamental group of a curve over a finite field and automorphic forms on GL(2), Amer. J. Math. 105(1983), 85-114.

[FP] Fimmel T., Parshin A. N., Introduction to the higher adelic theory, preprint, 1999.

[Fl] Flath D., Decomposition of representations into tensor products, Automorphic Forms, Representations and L-functions (Corvalis conference), Proc. Symp. Pure Math., Part 1, AMS, 33(1979), 179-184.

[F1] Frenkel E., Recent advances in the Langlands program, Bull. Amer. Math. Soc., 44(2004), 151-184 (arxiv:0303.074).

[F2] Frenkel E., Gauge theory and Langlands duality, Séminaire N. Bourbaki, expose 1010 , June 2009.

[GK] Ganter N., Kapranov M., Representations and character theory in 2categories, 2006 (arxiv:math0602510)

[G] Gelbart S., Elliptic curves and automorphic representantions, Adv. Math., 21(1976), 235-292.

[GJ] Godement R., Jacquet H. Zeta functions of simple algebras, Lecture Notes in Mathematics, Springer, 260(1972).

[HT] Harris M. and Taylor R., The geometry and cohomology of some simple Shimura varieties, Annals of Math. Studies 151, Princeton University Press, Princeton N.J., 2001.

[Has] Hasewinkel M., Corps de classes local. in Demazure M., Gabriel P., Groupes algébriques. Tome I., Paris, 1970.

[H] Henniart G., Une preuve simple des conjectures de Langlands pour $G L(n)$ sur un corps padique, Invent. Math. 139, 2000, 439-455.

[HH] Henniart G.,Herb R., Automorphic induction for GL(n) (over local nonarchimedean fields), Duke Math. J., 78(1995), 131-192.

[I] Ikeda I., Multi-Dimensional Langlands Functoriality Principle: Notes on M. M. Kapranov's Work, Series on Number Theory and Its Applications, 3, World Scientific, to appear.

[Inv] Invitation to higher local fields (Münster, 1999), (ed. I. Fesenko, M. Kurihara), Geom. Topol. Monogr., 3, Geom. Topol. Publ., Coventry, 2000, 304pp.(arXiv:math/0012131, abstract) 
[Iw] K. Iwasawa, A note on functions, Proc. Intern. Congres Math. (1950), Cambridge, Mass., 1950, vol.2, 322.

[J] Jacquet H. Principal L-functions of the linear group, Automorphic Forms, Representations and $L$-functions (Corvalis conference), Proc. Symp. Pure Math., Part 2, AMS, 33(1979), 63-86.

[JL] Jacquet H., Langlands R., Automorphic Forms on GL(2), Lecture Notes in Mathematics, 114(1970) (рус. пер. Жаке Э, Ленглендс Р. Автоморфные формы на GL(2), М., Мир, 1973).

[K] Kapranov M., Analogies between the Langlands correspondence and topological quantum field theory. Functional analysis on the eve of 21st century, Birkhäuser, 1995, vol. 1, 119-151.

[Ka] Kato K., Residue homomorphisms in Milnor's K-theory, Galois Groups and their Representations, Adv. Studies in Pure Math., 2(1983), 153172 .

[KT] Kawada Y., Tate J. On the Galois cohomology of unramified extensions of function fields in one variable, Amer. J. Math. 77(1955), 197-217.

[Kn] Knapp A. W. Local Langlands correspondance: the Archimedean case, Motives, Proc. Symp. Pure Math., AMS, 55(1994), Part 2, 393-410

[Koch] Koch H., Galoissche theorie der p-Erweiterungen, Berlin, 1970 (pyc. пер. Koх Х., Теория Галуа р-расширений, М., Мир, 1973).

[Ko] Koya Y., A generalization of class formations by using hypercohomology, Inven. Math., 101(1990), 705-715

[L] Lafforgue L., Chtoucas de Drinfeld et correspondance de Langlands, Invent. Math. 147(2002), 1-242.

[L1] Langlands R., Problems in the theory of automorphic forms, Lecture Notes in Mathematics, 170(1970) (рус. пер. Математика, 15:2, 1971, $57-83)$

[L2] Langlands R., Representations of abelian algebraic groups, circulated as a preprint from 1969 and published in Olga Taussky-Todd issue of Pacific J. of Math. 61(1988), 1-19.

[L3] Ленглендс P., Функториальность и взаимность. Курс лекций, Москва, МИАН, сентябрь-октябрь 2011 (http://publications.ias.edu/sites/default/files/lectures.pdf). 
[LRS] Laumon G., Rapoport M., Stuhler U., D-elliptic sheaves and the Langlands correspondence, Invent. Math. 113, 1993, 217-338.

[Liu] Liu D., Tame symbols and reciprocity laws on arithmetic surfaces, arXiv: 1203.6712.

[M] Morrow M., Grothendieck's trace map for arithmetic surfaces via residues and higher adeles, (arXiv:1101.1883)

[O1] Osipov D. V., Adelic constructions of direct images of differentials and symbols, Mat. Sb., 188:5 (1997), 59-84; English transl. in Sb. Math. 188:5 (1997), 697-723 (arXiv:9802.112).

[O2] Osipov D. V., Unramified two-dimensional Langlands correspondence, Izvestija RAN, ser. math., 77:4(2013)(arXiv:1210.3780).

[P1] Parshin A. N., Adeles, Chern classes and L-functions, Crelle J. 341(1983), 174-192.

[P2] Parshin A. N., Numbers as functions (developement of an idea in Moscow school of algebraic geometry), Nice talk, 1996. Mathemathical events of the XXth century, Springer-Phasis, Berlin-Moscow, 2006, 297329 (arXiv:0912.3785).

[P3] Parshin A. N., Representations of higher adelic groups and arithmetic, Proc. Intern. Congr. Math. Hyderabad, 2010, vol. 1, 362-392 (arXiv:1012.0486).

[P4] Parshin A. N.,Notes on the Poisson formula, St. Petersburg Math. J. 23:5 (2011), 781-818 (arXiv:1011.3392).

[Ram] Ramakrishnan D. Pure motives and automorphic forms, Motives, Proc. Symp. Pure Math., Part 2, AMS, 55(1994), 411-446.

[R] W. Raskind, Abelian class field theory of arithmetic schemes, (Jacob, Bill (ed.) et al.), $K$-theory and algebraic geometry: connections with quadratic forms and division algebras. Proc. Symp. Pure Math. 58, Part 1, Amer. Math. Soc., Providence, RI, 1995, 85-187.

[S1] Serre J.-P., Groupes algébriques et corps de classes, Paris, 1959 (pyc. пер. Серр Ж.-П., Алгебраические группы и поля классов, М., Мир, 1968).

[S2] Serre J.-P., Sur les corps locaux a corps residuel algébriquement clos Bull. Soc. Math. France, 89(1961), 105-154. 
[S3] Serre J.-P., Facteurs locaux des fonctions zêta des variétés algébriques (définitions et conjectures), Séminaire Delange-Pisot-Poitou, 1969/70, n 19, 11 mai 1970, 1-15 (рус. пер. Математика, 15:1, 1971, 3-13).

[Sh] Шафаревич И. P. [Shafarevich I. R.], Поля алгебраических чисел [Fields of algebraic numbers], Proc. Intern. Congr. Math. Stockholm 1962. Djursholm: Inst. Mittag-Lefler, 1963, 163-176. ICM Stockholm, 1962.

[T1] Tate J., Fourier analysis in number fields and Hecke's zeta-function, Thesis, Princeton, 1950 (published in Algebraic Number Theory, Ed. J. W. S. Cassels and A. Fröhlich, Academic Press, London-New York, 1967).

[T2] Tate J., Number-theoretical background, Proc. Symp. Pure Math., Corvalis conference, Part 2, AMS, 33(1979), 3-26. (рус.пер. Автоморфные формы, представления и $L$-функции, М., Мир, 1984, 73-112).

[W1] Weil A., Letter to Simone Weil (1940). Collected Works, Springer, New York-Heidelberg-Berlin, 1979, Vol. 1, 244-256; Notices AMS 52(2005), 334-341.

[W2] Weil A., Number-theory and algebraic geometry, Proc. Intern. Congr. Math. Cambridge, 1950, vol. 2, 90-102. (рус.пер. Математика, 2:4, 1958, 49-58).

[W3] Weil A., Zeta-functions and Mellin transforms, Proc. Bombay Coll. on Algebraic Geometry, TIFR, Bombay, 1968, 409-426.

[W4] Weil A., Basic number theory, Grundlehren Math. Wiss., Bd. 144, 3rd ed., Springer-Verlag, Berlin, 1974.

[Zh] Желобенко Д. П., Гармонический анализ на полупростых комплексных группах Ли, М., Наука, 1974 [ Zhelobenko D.P., Harmonic analysis on semisimple complex Lie groups, Current Problems of Mathematics, Nauka, Moscow 1974, 240 pp.]. .

Steklov Mathematical Institute,

Russian Academy of Sciences,

Russia 119991, Moscow, Gubkina str. 8

e-mail parshin@mi.ras.ru 